Bull. Soc. math. France

130 (3), 2002, p. $457-491$

HYPERIDEAL POLYHEDRA IN HYPERBOLIC 3-SPACE

\author{
By Xiliang BaO \& Francis Bonahon
}

\begin{abstract}
A hyperideal polyhedron is a non-compact polyhedron in the hyperbolic 3 -space $\mathbb{H}^{3}$ which, in the projective model for $\mathbb{H}^{3} \subset \mathbb{R P}^{3}$, is just the intersection of $\mathbb{H}^{3}$ with a projective polyhedron whose vertices are all outside $\mathbb{H}^{3}$ and whose edges all meet $\mathbb{H}^{3}$. We classify hyperideal polyhedra, up to isometries of $\mathbb{H}^{3}$, in terms of their combinatorial type and of their dihedral angles.

RÉSUmÉ (Polyèdres hyperidéaux de l'espace hyperbolique de dimension 3)

Un polyèdre hyperidéal est un polyèdre non-compact de l'espace hyperbolique $\mathbb{H}^{3}$ de dimension 3 qui, dans le modèle projectif pour $\mathbb{H}^{3} \subset \mathbb{R} \mathbb{P}^{3}$, est simplement l'intersection de $\mathbb{H}^{3}$ avec un polyèdre projectif dont les sommets sont tous en dehors de $\mathbb{H}^{3}$ et dont toutes les arêtes rencontrent $\mathbb{H}^{3}$. Nous classifions ces polyèdres hyperidéaux, à isométrie de $\mathbb{H}^{3}$ près, en fonction de leur type combinatoire et de leurs angles diédraux.
\end{abstract}

Consider a compact convex polyhedron $P$, intersection of finitely many halfspaces in one of the three $n$-dimensional homogeneous spaces, namely the euclidean space $\mathbb{E}^{n}$, the sphere $\mathbb{S}^{n}$ or the hyperbolic space $\mathbb{H}^{n}$. The boundary of $P$ inherits a natural cell decomposition, coming from the faces of the polyhedron.

Texte reçu le 26 juin 2001, accepté le 8 décembre 2001

Xiliang BaO, Department of Mathematics, University of Southern California, Los Angeles CA 90089-1113 (USA) • Computer Associates, 9740 Scranton Road \# 200, San Diego, CA 92121-1745 (USA) • E-mail : xiliang.bao@ca.com

Francis Bonahon, Department of Mathematics, University of Southern California, Los Angeles CA 90089-1113, (USA) • E-mail : fbonahon@math.usc.edu

Url : http://math.usc.edu/ fbonahon

2000 Mathematics Subject Classification. — 51M09.

Key words and phrases. - Hyperbolic space, polyhedron, ideal polyhedron, hyperideal.

This work was partially supported by NSF grants DMS-9504282 and DMS-9803445 from the US National Science Foundation. 
Along each $(n-2)$-face $e$, we can measure the internal dihedral angle $\left.\alpha_{e} \in\right] 0, \pi[$ between the two $(n-1)$-faces meeting along $e$. A natural question then arises: If we are given an $(n-1)$-dimensional cell complex $X$ with a weight $\left.\alpha_{e} \in\right] 0, \pi[$ attached to each $(n-2)$-dimensional cell $e$, is there a convex polyhedron $P$ in $\mathbb{E}^{n}, \mathbb{S}^{n}$ or $\mathbb{H}^{n}$ whose boundary has the combinatorial structure of this cell complex $X$, and such that $\alpha_{e}$ is the dihedral angle of $P$ along the face $e$ ?

An explicit computation provides a full answer in the simplest case where $X$ is the boundary of the $n$-simplex. The solution involves the signatures of various minors of the symmetric $n \times n$-matrix whose $i j$-entry is +1 if $i=j$ and is $-\cos \alpha_{e_{i j}}$, where $e_{i j}$ is the edge joining the $i$-th vertex to the $j$-th vertex, if $i \neq j$; see [6], [17]. In particular, the answer is expressed in terms of the signs of polynomials in $\cos \alpha_{e_{i j}}$. Since this condition on the angles $\alpha_{e}$ is not that easy to handle, one can expect the general case to be quite intractable, and this indeed seems to be the case.

In general, the main technical difficulty is to control the combinatorics as one deforms the polyhedron $P$. A typical problem occurs when a $p$-dimensional face becomes $(p-1)$-dimensional, for instance when two vertices collide so that a 1-dimensional face shrinks to one point.

One way to bypass this technical difficulty is to impose additional restrictions which will prevent such vertex collisions and face collapses. For instance, one can require that all dihedral angles $\alpha_{e}$ are acute, namely lie in the interval $\left.] 0, \frac{1}{2} \pi\right]$. In this context, Coxeter [6] proved that every acute angled compact convex polyhedron in the euclidean space $\mathbb{E}^{n}$ is an orthogonal product of euclidean simplices, possibly lower dimensional; this reduces the problem to the case of euclidean simplices, which we already discussed. Similarly, Coxeter also proved that every acute angled convex polyhedron in the sphere $\mathbb{S}^{n}$ is a simplex. The situation is more complex in the hyperbolic space $\mathbb{H}^{n}$ but, when $n=3$, Andreev was able to classify all acute angled compact convex polyhedra in $\mathbb{H}^{3}$ in terms of their combinatorics and their dihedral angles [2].

In hyperbolic space, another approach to prevent vertex collisions is to put these vertices infinitely apart, by considering (non-compact finite volume) ideal polyhedra, where all vertices sit on the sphere at infinity $\partial_{\infty} \mathbb{H}^{n}$ of $\mathbb{H}^{n}$. In [11], Rivin classifies all ideal polyhedra in $\mathbb{H}^{3}$ in terms of their combinatorics and of their dihedral angles. The case of acute angled polyhedra with some vertices at infinity had been earlier considered by Andreev [3].

In this paper, we propose to go one step further by considering polyhedra in $\mathbb{H}^{3}$ whose vertices are 'beyond infinity', and which we call hyperideal polyhedra.

These are best described in Klein's projective model for $\mathbb{H}^{3}$. Recall that, in this model, $\mathbb{H}^{3}$ is identified to the open unit ball in $\mathbb{R}^{3} \subset \mathbb{R P}^{3}$, that geodesics of $\mathbb{H}^{3}$ then correspond to the intersection of straight lines of $\mathbb{R}^{3}$ with $\mathbb{H}^{3}$, and that totally geodesic planes in $\mathbb{H}^{3}$ are the intersection of linear planes with $\mathbb{H}^{3}$. In this projective model $\mathbb{H}^{3} \subset \mathbb{R P}^{3}$, a hyperideal polyhedron is defined as the 
intersection $P$ of $\mathbb{H}^{3}$ with a compact convex polyhedron $P^{\text {Proj }}$ of $\mathbb{R P}^{3}$ with the following properties:

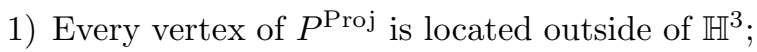

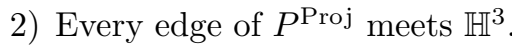

Note that we allow vertices of $P^{\text {Proj }}$ to be located on the unit sphere $\partial_{\infty} \mathbb{H}^{3}$ bounding $\mathbb{H}^{3}$, so that hyperideal polyhedra include ideal polyhedra as a special case.

From now on, we will restrict attention to the dimension $n=3$. Following the standard low-dimensional terminology, we will call vertex any 0-dimensional face or cell, an edge will be a 1-dimensional face or cell, and we will reserve the word face for any 2-dimensional face or cell.

To describe the combinatorics of a hyperideal polyhedron $P$, it is convenient to consider the dual graph $\Gamma$ of the cell decomposition of $\partial P$, namely the graph whose vertices correspond to the (2-dimensional) faces of $P$, and where two vertices $v$ and $v^{\prime}$ are connected by an edge exactly when the corresponding faces $f$ and $f^{\prime}$ of $P$ have an edge in common. Note that $\Gamma$ is also the dual graph of the projective polyhedron $P^{\text {Proj }}$ associated to $P$.

The graph $\Gamma$ must be planar, in the sense that it can be embedded in the sphere $\mathbb{S}^{2}$. In addition, $\Gamma$ is 3 -connected in the sense that it cannot be disconnected or reduced to a single point by removing 0,1 or 2 vertices and their adjacent edges; this easily follows from the fact that two distinct faces of $P^{\text {Proj }}$ can only meet along the empty set, one vertex or one edge. A famous theorem of Steinitz states that a graph is the dual graph of a convex polyhedron in $\mathbb{R}^{3}$ if and only if it is planar and 3-connected; see [8]. A classical consequence of 3connectedness is that the embedding of $\Gamma$ in $\mathbb{S}^{2}$ is unique up to homeomorphism of $\mathbb{S}^{2}$; see for instance $[9, \S 32]$. In particular, it intrinsically makes sense to talk of the components of $\mathbb{S}^{2}-\Gamma$. Note that these components of $\mathbb{S}^{2}-\Gamma$ naturally correspond to the vertices of $P^{\text {Proj }}$.

The results are simpler to state if, instead of the internal dihedral angle $\alpha_{e}$ of the polyhedron $P$ along the edge $e$, we consider the external dihedral angle $\left.\theta_{e}=\pi-\alpha_{e} \in\right] 0, \pi[$.

THEOREM 1. - Let $\Gamma$ be a 3-connected planar graph with a weight $\left.\theta_{e} \in\right] 0, \pi[$ attached to each edge e of $\Gamma$. There exists a hyperideal polyhedron $P$ in $\mathbb{H}^{3}$ with dual graph isomorphic to $\Gamma$ and with external dihedral angle $\theta_{e}$ along the edge corresponding to the edge e of $\Gamma$ if and only if the following two conditions are satisfied:

1) For every closed curve $\gamma$ embedded in $\Gamma$ and passing through the edges $e_{1}$, $e_{2}, \ldots, e_{n}$ of $\Gamma, \sum_{i=1}^{n} \theta_{e_{i}} \geq 2 \pi$ with equality possible only if $\gamma$ is the boundary of a component of $\mathbb{S}^{2}-\Gamma$;

2) For every arc $\gamma$ embedded in $\Gamma$, passing through the edges $e_{1}, e_{2}, \ldots, e_{n}$ of $\Gamma$, joining two distinct vertices $v_{1}$ and $v_{2}$ which are in the closure of the same 
component $A$ of $\mathbb{S}^{2}-\Gamma$ but such that $\gamma$ is not contained in the boundary of $A$, $\sum_{i=1}^{n} \theta_{e_{i}}>\pi$.

In addition, for the projective polyhedron $P^{\mathrm{Proj}}$ associated to $P$, a vertex of $P^{\text {Proj }}$ is located on the sphere at infinity $\partial_{\infty} \mathbb{H}^{3}$ if and only if equality holds in Condition 1 for the boundary of the corresponding component of $\mathbb{S}^{2}-\Gamma$.

Note that Theorem 1 generalizes Rivin's existence result for ideal polyhedra [11].

Theorem 2. - The hyperideal polyhedron $P$ in Theorem 1 is unique up to isometry of $\mathbb{H}^{3}$.

Theorem 2 was proved by Rivin [10], [11] for ideal polyhedra, and by Rivin and Hodgson [12] (if we use the truncated polyhedra discussed in $\S 1$ ) for the other extreme, namely for hyperideal polyhedra with no vertex on the sphere at infinity. Even in these cases, one could argue that our proof is a little simpler, as it is based on relatively simple infinitesimal lemmas followed by covering space argument, as opposed to the more delicate global argument of Lemma 4.11 of [12]. However, the main point of Theorem 2 is that it is a key ingredient for the proof of Theorem 1, justifying once again the heuristic principle that "uniqueness implies existence". Theorem 2 is the reason why we introduced Condition 2 in the definition of hyperideal polyhedra, as it fails for general polyhedra without vertices in $\mathbb{H}^{3}$.

Our proof of Theorems 1 and 2 is based on the continuity method pioneered by Aleksandrov [1] and further exploited in [2] and [12]. We first use an implicit function theorem, proved through a variation of Cauchy's celebrated rigidity theorem for euclidean polyhedra [5], to show that a hyperideal polyhedron is locally determined by its combinatorial type and its dihedral angles. We then go from local to global by a covering argument.

Although the generalization of the results of [11] from ideal polyhedra to hyperideal polyhedra is of interest by itself, the real motivation for this work was to provide a proof of the classification of ideal polyhedra which locally controls the combinatorics of the polyhedra involved. Ideal polyhedra play an important role in 3-dimensional geometry, as they can be used as building blocks to construct hyperbolic 3-manifolds through the use of ideal triangulations, possibly not locally finite. To study deformations of a hyperbolic metric on a 3-manifold, it is therefore useful to have a good classification of the deformations of ideal polyhedra within a given combinatorial type. Unfortunately, Rivin's argument in [11] is indirect. He first uses the classification of compact hyperbolic polyhedra by their dual polyhedra [12], where one completely looses control of the combinatorics, and he extends it to ideal polyhedra by passing to the limit as the vertices go to infinity; he then observes that for ideal polyhedra the dual polyhedron does determine the combinatorics of the ideal polyhedron.

TOME $130-2002-\mathrm{N}^{\mathrm{O}} 3$ 
In this regard, the local characterization of hyperideal polyhedra by their dihedral angles provided by our Theorem 11, which is already the key technical step in this paper, may be its most useful result for applications.

The paper [10] provides a different approach to a local control of ideal polyhedra through their combinatorics and dihedral angles. The reader is also referred to [13], [14] for the consideration of other rigidity properties of polyhedra in hyperbolic 3 -space.

It may also be of interest that Theorems 1 and 2 can be translated into purely euclidean (or at least projective) statements. Indeed, they provide a classification of hyperideal projective polyhedra $P^{\text {Proj }}$ modulo the action of the group $\mathrm{PO}(3,1)$ consisting of those projective transformations of $\mathbb{R P}^{3}$ that respect the unit sphere $\mathbb{S}^{2} \subset \mathbb{R}^{3} \subset \mathbb{R} \mathbb{P}^{3}$. This is particularly remarkable when one notices that, for an edge $e$ of $P=\mathbb{H}^{3} \cap P^{\text {Proj }}$, the hyperbolic dihedral angle $\theta_{e}$ of $P$ is equal to the euclidean angle between the two circles $\Pi \cap \mathbb{S}^{2}$ and $\Pi^{\prime} \cap \mathbb{S}^{2}$ at their intersection points, where $\Pi$ and $\Pi^{\prime}$ are the two euclidean planes respectively containing the two faces of $P$ that meet along $e$. By duality, Theorems 1 and 2 also classify convex projective polyhedra whose faces all meet the unit sphere $\mathbb{S}^{2} \subset \mathbb{R}^{3} \subset \mathbb{R} \mathbb{P}^{3}$ but whose edges are all disjoint from the closed ball $\mathbb{H}^{3} \cup \mathbb{S}^{2}$, modulo the action of $\mathrm{PO}(3,1)$.

Theorems 1 and 2 for the somewhat simpler case of strictly ideal polyhedra, where all vertices of $P^{\text {Proj }}$ are outside of the closure of $\mathbb{H}^{3}$, appeared in [4]. The final draft of this paper was essentially completed while the second author was visiting the Institut des Hautes Études Scientifiques, which he would like to thank for its productive hospitality. The authors are also grateful to the referee for several suggestions of improvement of the exposition, including a simplification of the proof of Proposition 6.

\section{Hyperideal polyhedra}

We first recall a few basic facts about the projective model for $\mathbb{H}^{3}$ (see for instance [16]).

Consider the symmetric bilinear form

$$
B\left(\left(X_{0}, X_{1}, X_{2}, X_{3}\right),\left(Y_{0}, Y_{1}, Y_{2}, Y_{3}\right)\right)=-X_{0} Y_{0}+X_{1} Y_{1}+X_{2} Y_{2}+X_{3} Y_{3}
$$

on $\mathbb{R}^{4}$. In the projective space $\mathbb{R} \mathbb{P}^{3}$, we consider the image $\mathbb{H}^{3}$ of the set of those $X \in \mathbb{R}^{4}$ with $B(X, X)<0$. For the standard embedding of $\mathbb{R}^{3}$ in $\mathbb{R P}^{3}$, defined by associating to $\left(x_{1}, x_{2}, x_{3}\right) \in \mathbb{R}^{3}$ the point of $\mathbb{R} \mathbb{P}^{3}$ with homogeneous coordinates $\left(1, x_{1}, x_{2}, x_{3}\right)$, the subset $\mathbb{H}^{3}$ just corresponds to the open unit ball in $\mathbb{R}^{3}$.

The projection $\mathbb{R}^{4} \rightarrow \mathbb{R P}^{3}$ induces a diffeomorphism between $\mathbb{H}^{3}$ and the set $H$ of those $X=\left(X_{0}, X_{1}, X_{2}, X_{3}\right) \in \mathbb{R}^{4}$ with $B(X, X)=-1$ and $X_{0}>0$. The tangent space $T_{X} H$ of $H$ at $X$ is equal to the $B$-orthogonal of $X$ and, 
since $B(X, X)<0$ and $B$ has signature $(3,1)$, the restriction of $B$ to $T_{X} S$ is therefore positive definite. The corresponding riemannian metric on $H \cong \mathbb{H}^{3}$ is exactly the hyperbolic metric of the projective model for $\mathbb{H}^{3}$.

The group $\mathrm{O}(3,1)$ of linear $B$-isometries of $\mathbb{R}^{4}$ induces an action of $\mathrm{PO}(3,1)=\mathrm{O}(3,1) /\{ \pm \mathrm{Id}\}$ on $\mathbb{H}^{3}$ which respects the metric of $\mathbb{H}^{3}$. The group $\mathrm{PO}(3,1)$ is actually equal to the whole isometry group of $\mathbb{H}^{3}$. Note that $\mathrm{PO}(3,1)$ is naturally isomorphic to the subgroup of $\mathrm{O}(3,1)$ consisting of those elements that respect $H$.

In this model, the geodesics of $\mathbb{H}^{3}$ are exactly the non-empty intersections of $\mathbb{H}^{3}$ with projective lines of $\mathbb{R P}^{3}$ or, equivalently, with straight lines of $\mathbb{R}^{3} \subset \mathbb{R} \mathbb{P}^{3}$. Similarly, the (totally geodesic) hyperbolic planes in $\mathbb{H}^{3}$ correspond to the nonempty intersections of $\mathbb{H}^{3}$ with projective planes of $\mathbb{R P}^{3}$ or, equivalently, with affine planes of $\mathbb{R}^{3}$. We will occasionally use the property that, given two projective planes $\Pi$ and $\Pi^{\prime}$ whose intersection meets $\mathbb{H}^{3}$, the hyperbolic dihedral angle between the hyperbolic planes $\Pi \cap \mathbb{H}^{3}$ and $\Pi^{\prime} \cap \mathbb{H}^{3}$ along the geodesic $\Pi \cap \Pi^{\prime} \cap \mathbb{H}^{3}$ is exactly equal to the euclidean angle between the circles $\Pi \cap \partial_{\infty} \mathbb{H}^{3}$ and $\Pi^{\prime} \cap \partial_{\infty} \mathbb{H}^{3}$ at their two intersection points in the sphere at infinity $\partial_{\infty} \mathbb{H}^{3}$ bounding $\mathbb{H}^{3}$ in $\mathbb{R}^{3}$.

One of the most valuable features of the projective model for $\mathbb{H}^{3}$ is its duality properties. Given a $k$-dimensional projective subspace $\ell \subset \mathbb{R P}^{3}$, with $0 \leq k \leq 2$, the $B$-orthogonal $L^{\perp}$ of the $(k+1)$-dimensional linear subspace $L$ of $\mathbb{R}^{4}$ corresponding to $\ell$ is a $(3-k)$-dimensional linear subspace of $\mathbb{R}^{4}$, and therefore defines a $(2-k)$-dimensional projective space $\ell^{\perp}$ of $\mathbb{R P}^{3}$.

In particular, if $x$ is a point which is not in the closure of $\mathbb{H}^{3}$ in $\mathbb{R P}^{3}, x^{\perp}$ is a plane which must intersect $\mathbb{H}^{3}$, since otherwise $B$ would be positive definite or degenerate. By elementary linear algebra, if $y \in x^{\perp} \cap \mathbb{H}^{3}$ and if $l$ denotes the projective line passing through $x$ and $y$, the geodesic $l \cap \mathbb{H}^{3}$ is orthogonal to the hyperbolic plane $x^{\perp} \cap \mathbb{H}^{3}$ for the metric of $\mathbb{H}^{3}$. Similarly, if $z$ belongs to the intersection of $x^{\perp}$ with the sphere $\partial_{\infty} \mathbb{H}^{3}$ bounding $\mathbb{H}^{3}$, then the projective line joining $x$ to $z$ cannot meet $\mathbb{H}^{3}$, and is therefore tangent to $\partial_{\infty} \mathbb{H}^{3}$. This last point gives us a very geometric way to construct $x^{\perp}$ : Draw all the projective lines passing through $x$ and tangent to $\partial_{\infty} \mathbb{H}^{3}$; then $x^{\perp}$ is the projective plane which intersects $\partial_{\infty} \mathbb{H}^{3}$ along the circle formed by all the points of tangency.

When $x$ is a point of the sphere $\partial_{\infty} \mathbb{H}^{3}$, the dual plane $x^{\perp}$ is just the plane tangent to $\partial_{\infty} \mathbb{H}^{3}$ at $x$. This can, for instance, be seen by continuity from the previous case.

Conversely, if $\Pi$ is a hyperbolic plane in $\mathbb{H}^{3}$, the point $x$ such that $\Pi=$ $x^{\perp} \cap \mathbb{H}^{3}$ is necessarily outside of the closure of $\mathbb{H}^{3}$ in $\mathbb{R} P^{3}$, for signature reasons. In particular, the line $L$ in $\mathbb{R}^{4}$ corresponding to $x \in \mathbb{R P}^{3}$ contains exactly two vectors $X \in \mathbb{R}^{4}$ with $B(X, X)=+1$. If, in addition, $\Pi$ is endowed with a transverse orientation, this defines a transverse orientation for the linear subspace $L^{\perp} \subset \mathbb{R}^{4}$. By definition, the unit normal vector of the transversely 
oriented hyperbolic plane $\Pi$ is the vector $X \in L$ with $B(X, X)=+1$ and which crosses $L^{\perp}$ in the direction of this transverse orientation.

Now, consider a hyperideal polyhedron $P$ in $\mathbb{H}^{3}$, as defined in the introduction. Recall that this means that $P=P^{\operatorname{Proj}} \cap \mathbb{H}^{3}$, where $P^{\text {Proj }}$ is a convex projective polyhedron in $\mathbb{R P}^{3}$ whose vertices are all outside of $\mathbb{H}^{3}$ and whose edges all meet $\mathbb{H}^{3} \cup \partial_{\infty} \mathbb{H}^{3}$. A vertex of $P^{\text {Proj }}$ which is on the sphere at infinity $\partial_{\infty} \mathbb{H}^{3}$ is said to be ideal; otherwise, it is strictly hyperideal.

At this point, it may be useful to remind the reader that a convex projective polyhedron in $\mathbb{R P}^{3}$ is the closure of a component of the complement of finitely many projective planes in $\mathbb{R P}^{3}$. To avoid degenerate cases, we require in addition that a convex projective polyhedron does not contain any projective line or, equivalently, that it is disjoint from at least one projective plane in $\mathbb{R P}^{3}$, so that is is homeomorphic to a closed 3-ball.

Since every edge of $P^{\operatorname{Proj}}$ meets $\mathbb{H}^{3}$, so does every face and it follows that

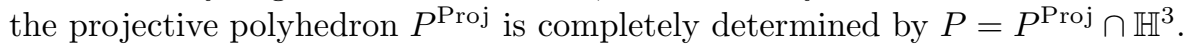

For future reference, we note the following easy property.

Lemma 3. - For any two distinct vertices $v$ and $v^{\prime}$ of the projective polyhedron $P^{\text {Proj }}$ associated to the hyperideal polyhedron $P$, the line segment joining $v$ to $v^{\prime}$ in $P^{\mathrm{Proj}}$ meets $\mathbb{H}^{3}$.

Proof. - Modifying $P$ with an element of $\mathrm{PO}(3,1)$ if necessary, we can assume that $P^{\text {Proj }} \subset \mathbb{R}^{3} \subset \mathbb{R P}^{3}$ without loss of generality. Then, consider the radial projection $\mathbb{R}^{3}-\{v\} \rightarrow \mathbb{S}^{2}$ to the unit sphere centered at $v$. The image $\varphi\left(\mathbb{H}^{3}\right)$ is a spherical open disk contained in a hemisphere of $\mathbb{S}^{2}$, and $\varphi(P)$ is a convex spherical polygon whose vertices are contained in $\varphi\left(\mathbb{H}^{3}\right)$. It follows that $\varphi(P)$ is contained in $\varphi\left(\mathbb{H}^{3}\right)$, by convexity. In particular, $\varphi\left(v^{\prime}\right)$ is in $\varphi\left(\mathbb{H}^{3}\right)$, and it follows that the line segment $v v^{\prime}$ must meet $\mathbb{H}^{3}$.

Consider a strictly hyperideal vertex $v$ of $P^{\text {Proj }}$. Among the two closed hyperbolic half-spaces delimited by the hyperbolic plane $v^{\perp} \cap \mathbb{H}^{3}$ in $\mathbb{H}^{3}$, let $H_{v}$ be the one with the following property: For every other vertex $v^{\prime} \neq v$ of $P^{\text {Proj, }}$ the (unique) oriented line segment $k$ of $\mathbb{R P}^{3}$ which goes from $v$ to $v^{\prime}$ in $P^{\text {Proj }}$ exits $H_{v}$ at its intersection point with $v^{\perp} \cap \mathbb{H}^{3}$. (To make sure that this really makes sense, it may be useful to modify $P^{\operatorname{Proj}}$ by an element of $\mathrm{PO}(3,1)$ so that it is contained in $\mathbb{R}^{3} \subset \mathbb{R} P^{3}$ and to use the geometric description of $v^{\perp} \cap \mathbb{H}^{3}$ by lines passing through $v$ and tangent to $\partial_{\infty} \mathbb{H}^{3}$.) In other words, the halfspace $H_{v}$ is on the same side of $x^{\perp} \cap \mathbb{H}^{3}$ as $v$ with respect to the other vertices of $P^{\text {Proj }}$

Lemma 4. - For any two distinct hyperideal vertices $v, v^{\prime}$ of $P^{\mathrm{Proj}}$, the associated hyperbolic half-spaces $H_{v}$ and $H_{v^{\prime}}$ in $\mathbb{H}^{3}$ are disjoint.

Proof. - By definition of $H_{v}$ and $H_{v^{\prime}}$, it clearly suffices to show that the two hyperbolic planes $v^{\perp} \cap \mathbb{H}^{3}$ and $v^{\prime \perp} \cap \mathbb{H}^{3}$ are disjoint. In $\mathbb{R P}^{3}$, the intersection 
of $v^{\perp}$ and $v^{\prime \perp}$ is the line $\left(v v^{\prime}\right)^{\perp}$ dual to the line $v v^{\prime}$ passing through $v$ and $v^{\prime}$. By Lemma 3, the line $v v^{\prime}$ meets $\mathbb{H}^{3}$ and its dual line $\left(v v^{\prime}\right)^{\perp}$ is consequently disjoint from the closure of $\mathbb{H}^{3}$. It follows that the two hyperbolic planes $v^{\perp} \cap \mathbb{H}^{3}$ and $v^{\prime \perp} \cap \mathbb{H}^{3}$ are disjoint, and therefore that $H_{v}$ and $H_{v^{\prime}}$ are also disjoint.

If $v$ is an ideal vertex of $P^{\text {Proj }}$, we choose a small horoball $H_{v}$ centered at $v$. In the projective model $\mathbb{H}^{3} \subset \mathbb{R}^{3} \subset \mathbb{R P}^{3}, H_{v}$ is just the intersection with $\mathbb{H}^{3}$ of a closed euclidean ball which is contained in $\mathbb{H}^{3} \cup \partial_{\infty} \mathbb{H}^{3}$ and tangent to $\partial_{\infty} \mathbb{H}^{3}$ at $v$. There are of course many possible choices for such a horoball, but we choose it so that all $H_{v}$ thus associated to strictly hyperideal and ideal vertices are pairwise disjoint.

By definition, the truncated polyhedron $P^{\text {Trun }}$ associated to the hyperideal polyhedron $P$ is obtained by removing from $P$ its intersection with the interior of each such $H_{v}$. Note that $P^{\text {Trun }}$ is completely determined if all the vertices of $P^{\text {Proj }}$ are strictly hyperideal, and defined modulo choice of the horoballs $H_{v}$ associated to the ideal vertices in the general case.

This truncated polyhedron $P^{\text {Trun }}$ will play an important rôle in our arguments. By construction, $P^{\text {Trun }}$ is compact. Its faces are of two types. The first type of face is the intersection of a face of $P$ with $P^{\text {Trun }}$. The second type of face is the intersection of $P^{\text {Trun }}$ with one of the $\partial H_{v}$. This second type can itself be subdivided in two subtypes, according to whether $\partial H_{v}$ is a horosphere, when the vertex $v$ is ideal, or a hyperbolic plane, when the vertex $v$ is strictly hyperideal.

\section{Necessary conditions}

Proposition 5. - Let $\Gamma$ be the dual graph of a hyperideal polyhedron $P$ in $\mathbb{H}^{3}$ and, for every edge e of $\Gamma$, let $\left.\theta_{e} \in\right] 0, \pi[$ be the external dihedral angle of $P$ at the edge corresponding to $e$. For every closed curve $\gamma$ embedded in $\Gamma$ and passing through the edges $e_{1}, e_{2}, \ldots, e_{n}$ of $\Gamma$, then $\sum_{i=1}^{n} \theta_{e_{i}} \geq 2 \pi$ with equality if only if $\gamma$ is the boundary of a component of $\mathbb{S}^{2}-\Gamma$ corresponding to an ideal vertex of $P^{\text {Proj. }}$.

Proof. - Counting indices modulo $n$, let $v_{i}$ be the vertex of $\Gamma$ that is between $e_{i-1}$ and $e_{i}$ in $\gamma$, and let $f_{i}$ be the face of $P$ corresponding to $v_{i}$. Let $\Pi_{i} \subset \mathbb{H}^{3}$ be the hyperbolic plane containing $f_{i}$, and let $H_{i} \subset \mathbb{H}^{3}$ be the half-space delimited by $\Pi_{i}$ and containing $P$. Let $P^{\prime}$ be the intersection of the $H_{i}$. The boundary $\partial P^{\prime}$ is an annulus, and is decomposed as a union of infinite strips $\Pi_{i} \cap P^{\prime}$, in such a way that $\Pi_{i} \cap P^{\prime}$ and $\Pi_{i+1} \cap P^{\prime}$ meet along the geodesic of $\mathbb{H}^{3}$ containing the edge of $P$ corresponding to $e_{i}$.

The closure of $P^{\prime}$ in $\mathbb{H}^{3} \cup \partial_{\infty} \mathbb{H}^{3}$ meets $\partial_{\infty} \mathbb{H}^{3}$ along two topological disks $D_{1}$ and $D_{2}$, one of which will be reduced to a single point if (and only if) $\gamma$ is the boundary of a component of $\mathbb{S}^{2}-\Gamma$ which corresponds to an ideal vertex

TOME $130-2002-\mathrm{N}^{\mathrm{O}} 3$ 
of $P$. The disk $D_{j}$ is the union of circle $\operatorname{arcs} k_{1}^{j}, k_{2}^{j}, \ldots, k_{n}^{j}$, where each $k_{i}^{j}$ is contained in the circle $C_{i} \subset \partial_{\infty} \mathbb{H}^{3}$ bounding the hyperbolic plane $H_{i}$. Note that, at the point where $k_{i}^{j}$ meets $k_{i+1}^{j}$, the euclidean external angle between these two arcs is equal to the angle between the circles $C_{i}$ and $C_{i+1}$, namely to the hyperbolic external dihedral angle $\theta_{e_{i}}$ of $P$ along the edge $e_{i}$. This holds even when $k_{i}^{j}$ or $k_{i+1}^{j}$ is reduced to a point, provided we use the convention that the tangent line at a point of $k_{i}^{j}$ is equal to the tangent line of $C_{i}$ at the same point.

First consider the case where neither one of the two disks $D_{1}$ and $D_{2}$ is reduced to a point. Let $\varphi: \partial_{\infty} \mathbb{H}^{3} \rightarrow \mathbb{R}^{2}$ be defined by stereographic projection from a point in the interior of $D_{2}$. Because the stereographic projection sends circle to circle and because this stereographic projection was performed from a point in the interior of $D_{2}$, the $\operatorname{arcs} \varphi\left(k_{i}^{1}\right)$ forming the boundary of $\varphi\left(D_{1}\right)$ are circle arcs whose curvature vectors point away from $\varphi\left(D_{1}\right)$. In particular, outside of the corners of $\partial \varphi\left(D_{1}\right)$, the geodesic curvature $\kappa$ of $\partial \varphi\left(D_{1}\right)$ is negative. Also, because the stereographic projection preserves angles, the angle between $\varphi\left(k_{i}^{1}\right)$ and $\varphi\left(k_{i+1}^{1}\right)$ is equal to the angle between $k_{i}^{1}$ and $k_{i+1}^{1}$, namely to $\theta_{e_{i}}$. If we apply to $\varphi\left(D_{1}\right)$ the Gauss-Bonnet formula (also known in this case as the Theorem of the Turning Tangents), $2 \pi=\int_{\partial \varphi\left(D_{1}\right)} \kappa+\sum_{i=1}^{n} \theta_{e_{i}}<\sum_{i=1}^{n} \theta_{e_{i}}$ where the inequality is strict because of our assumption that $D_{1}$ is not reduced to a single point. This proves the expected result in this case.

If one of $D_{1}$ and $D_{2}$, say $D_{2}$, is reduced to a point, consider again the stereographic projection $\varphi: \partial_{\infty} \mathbb{H}^{3} \rightarrow \mathbb{R}^{2}$ from this point of $D_{2}$. The sides $\varphi\left(k_{i}^{1}\right)$ of the polygon $\varphi\left(D_{1}\right)$ are now straight arcs, and the Gauss Bonnet formula gives $2 \pi=\sum_{i=1}^{n} \theta_{e_{i}}$ in this case.

Proposition 6. - Let $\Gamma$ be the dual graph of a hyperideal polyhedron $P$ in $\mathbb{H}^{3}$ and, for every edge e of $\Gamma$, let $\left.\theta_{e} \in\right] 0, \pi[$ be the dihedral angle of $P$ at the edge corresponding to $e$. For every arc $\gamma$ embedded in $\Gamma$, passing through the edges $e_{1}, e_{2}, \ldots, e_{n}$ of $\Gamma$, and joining two distinct vertices $v_{1}$ and $v_{n+1}$ which are in the closure of the same component $A$ of $\mathbb{S}^{2}-\Gamma$ but such that $\gamma$ is not contained in the boundary of $A$, then $\sum_{i=1}^{n} \theta_{e_{i}}>\pi$.

Proof. - It clearly suffices to restrict attention to those $\gamma$ which meet the closure of $A$ only at their two end points. Let $v$ be the vertex of $P^{\text {Proj }}$ corresponding to $A$.

If the vertex $v$ is on the sphere at infinity $\partial_{\infty} \mathbb{H}^{3}$ then, by Proposition 5 , we can join the two end points of $\gamma$ by an arc $\gamma^{\prime}$ in $\partial A$ crossing edges $e_{n+1}$, $\ldots, e_{n+p}$ such that $\sum_{i=n+1}^{n+p} \theta_{e_{i}} \leq \pi$. Applying Proposition 5 to the simple closed curve $\gamma \cup \gamma^{\prime}$, we obtain that $\sum_{i=1}^{n} \theta_{e_{i}} \geq \sum_{i=1}^{n+p} \theta_{e_{i}}-\pi \geq 2 \pi-\pi=\pi$. In addition, equality can occur only when $\gamma \cup \gamma^{\prime}$ is the boundary of a component $A^{\prime}$ of $\mathbb{S}^{2}-\Gamma$, which is different from $A$ since $\gamma$ is not contained in the boundary of $A$. In this case, $\gamma^{\prime}$ is contained in $\partial A \cap \partial A^{\prime}$ and therefore consists of a single 
edge $e_{n+1}$, so that $\sum_{i=n+1}^{n+p} \theta_{e_{i}}=\theta_{e_{n+1}}<\pi$. As a consequence, the inequality is always strict.

Now consider the other case, where the vertex $v$ is strictly hyperideal. Let $\rho$ be the hyperbolic reflection across the plane $v^{\perp}$, and let $P^{\prime}$ be the union $P \cup \rho(P)$. Because $v^{\perp}$ separates $v$ from the other vertices of $P^{\text {Proj }}$ and is orthogonal to the edges and faces of $P$ that it meets, $P^{\prime}$ is a hyperideal polyhedron. Each edge of $P^{\prime}$ is, either an edge of $P$ that does not meet $v^{\perp}$, or the image under $\rho$ of an edge of $P$ that does not meet $v^{\perp}$, or an edge of $P$ that meets $v^{\perp}$. Similarly, a face of $P^{\prime}$ is, either a face of $P$ that does not meet $v^{\perp}$, or the image under $\rho$ of a face of $P$ that does not meet $v^{\perp}$, or the intersection of a face of $P$ that meets $v^{\perp}$ with its image under $\rho$. As a consequence, the dual graph $\Gamma^{\prime}$ of $P^{\prime}$ is obtained by gluing together two copies of $\Gamma$ along the two images of $\partial A$.

Because of our assumption that $\gamma$ meets $\partial A$ only at its end points, the images of $\gamma$ in each of the two copies of $\Gamma$ form a closed curve $\gamma^{\prime}$ embedded in $\Gamma^{\prime}$. Applying Proposition 5 to the closed curve $\gamma^{\prime}$ in the graph $\Gamma^{\prime}$ dual to $P^{\prime}$, we conclude that $2 \sum_{i=1}^{n} \theta_{e_{i}}>2 \pi$, which concludes the proof.

LemMA 7. - Let $P$ be a convex cone with non-empty interior in $\mathbb{H}^{3}$, intersection of finitely many half-spaces each containing the point $x \in \mathbb{H}^{3}$ in its boundary. If $e_{1}, \ldots, e_{n}$ are the edges of $P$ and if $\left.\theta_{e} \in\right] 0, \pi[$ denotes the external dihedral angle of $P$ along the edge $e$, then $\sum_{i=1}^{n} \theta_{e_{i}}<2 \pi$.

Proof. - In the unit tangent sphere $T_{x}^{1} \mathbb{H}^{3}$ of $\mathbb{H}^{3}$ at $x$, consider the set $Q$ of those vectors which point towards $P$. Then $Q$ is a convex polygon with geodesic sides in the sphere $T_{x}^{1} \mathbb{H}^{3}$. In addition, the external angle of $Q$ at each of its corners is equal to the external dihedral angle of $P$ at the corresponding edge. The inequality then follows by application of the Gauss-Bonnet formula to $Q$.

\section{Spaces of polyhedra}

If $\Gamma$ is a planar 3 -connected graph, let $\widetilde{\mathcal{P}}_{\Gamma}$ denote the space of hyperideal polyhedra $P$ whose dual graph $\Gamma_{P}$ is identified to $\Gamma$. More precisely, an element of $\widetilde{\mathcal{P}}_{\Gamma}$ is a pair consisting of a hyperideal polyhedron $P$ and of an isomorphism $\Gamma \rightarrow \Gamma_{P}$ between $\Gamma$ and the dual graph $\Gamma_{P}$ of $P$.

Because working with $\Gamma$ often leads to confusing terminology (vertices of $\Gamma$ correspond to faces of the corresponding polyhedra, while vertices of the projective polyhedra correspond to components of $\mathbb{S}^{2}-\Gamma$, etc. . ), it is convenient to fix a hyperideal polyhedron $P_{0} \in \widetilde{\mathcal{P}}_{\Gamma}$, with associated projective polyhedron $P_{0}^{\text {Proj }}$. We will see in $\S 6$ that $\widetilde{\mathcal{P}}_{\Gamma}$ is always non-empty, but what is important here is that the combinatorics of $P_{0}$ can be abstractly described in terms of $\Gamma$. In particular, the set $F$ of faces of $P_{0}$ and $P_{0}^{\mathrm{Proj}}$ is naturally identified to the set of

TOME $130-2002-\mathrm{N}^{\mathrm{O}} 3$ 
vertices of $\Gamma$, and the set $V$ of vertices of $P_{0}^{\text {Proj }}$ is naturally identified to the set of components of $\mathbb{S}^{2}-\Gamma$. Recall that, because $\Gamma$ is 3 -connected, its embedding in $\mathbb{S}^{2}$ is unique up to homeomorphism of $\mathbb{S}^{2}$ (see for instance $[9, \S 32]$ ), so that these two sets $F$ and $V$ only depend on $\Gamma$. For every $P \in \widetilde{\mathcal{P}}_{\Gamma}$ with associated projective polyhedron $P^{\text {Proj }}$, the sets of faces and vertices of $P^{\text {Proj }}$ are now naturally identified to $F$ and $V$.

We endow $\widetilde{\mathcal{P}}_{\Gamma}$ with the topology induced by the embedding $\Phi: \widetilde{\mathcal{P}}_{\Gamma} \rightarrow\left(\mathbb{R P}^{3}\right)^{V}$ that associates to $P \in \widetilde{\mathcal{P}}_{\Gamma}$ the vertices of its associated projective polyhedron $P^{\text {Proj }}$

The group $\mathrm{PO}(3,1)$ has a natural action on $\widetilde{\mathcal{P}}_{\Gamma}$. Consider the quotient space $\mathcal{P}_{\Gamma}=\widetilde{\mathcal{P}}_{\Gamma} / \mathrm{PO}(3,1)$.

LEMMA 8. - The space $\mathcal{P}_{\Gamma}$ is Hausdorff.

Proof. - We need to show that, whenever two sequences $P_{n} \in \widetilde{\mathcal{P}}_{\Gamma}$ and $g_{n} \in$ $\mathrm{PO}(3,1)$ are such that $P_{n}$ converges to some $P \in \widetilde{\mathcal{P}}_{\Gamma}$ and $g_{n} P_{n}$ converges to some $Q \in \widetilde{\mathcal{P}}_{\Gamma}$, there is a $g \in \mathrm{PO}(3,1)$ such that $g P=Q$.

Pick four distinct faces $f_{0}, f_{1}, f_{2}, f_{3} \in F$ of $P_{0}^{\text {Proj }}$ in such a way that $f_{0}$ is adjacent to the faces $f_{1}, f_{2}$ and $f_{3}$. For each $i$, consider the hyperbolic plane that contains the face of $P$ corresponding to $f_{i}$, transversely oriented with the outward boundary orientation from $P$, and let $X_{i} \in \mathbb{R}^{4}$ be its unit normal vector, as defined in $\S 1$. A consequence of the choice of the faces $f_{0}, f_{1}, f_{2}, f_{3}$ is that the intersection of the four projective planes $X_{0}^{\perp}, X_{1}^{\perp}, X_{2}^{\perp}, X_{3}^{\perp}$ in $\mathbb{R P}^{3}$ is empty. It follows that the vectors $X_{0}, X_{1}, X_{2}, X_{3}$ are linearly independent, and form a basis $\left\{X_{0}, X_{1}, X_{2}, X_{3}\right\}$ for $\mathbb{R}^{4}$.

Similarly, the polyhedron $Q \in \widetilde{\mathcal{P}}_{\Gamma}$ provides a basis $\left\{Y_{0}, Y_{1}, Y_{2}, Y_{3}\right\}$ for $\mathbb{R}^{4}$, where $Y_{i} \in \mathbb{R}^{4}$ is the unit normal vector to the hyperbolic plane which contains the face of $Q$ corresponding to $f_{i}$, transversely oriented with the outward boundary orientation from $Q$, and the polyhedron $P_{n} \in \widetilde{\mathcal{P}}_{\Gamma}$ gives a similar basis $\left\{X_{0}^{n}, X_{1}^{n}, X_{2}^{n}, X_{2}^{n}\right\}$.

Since $P_{n}$ converges to $P$ for the topology of $\widetilde{\mathcal{P}}_{\Gamma}$, each vertex of $P_{n}^{\text {Proj }}$ converges to the corresponding vertex of $P^{\text {Proj }}$, and each of its faces consequently converges to the corresponding face of $P^{\text {Proj }}$. In particular, each $X_{i}^{n}$ converges to $X_{i}$ in $\mathbb{R}^{4}$. Similarly, since $g_{n} P_{n}$ converges to $Q$ in $\widetilde{\mathcal{P}}_{\Gamma}$, each $g_{n} X_{i}^{n}$ converges to $Y_{i}$. If $A_{n}$ denotes the matrix of $g_{n}$ from $\mathbb{R}^{4}$ with the basis $\left\{X_{0}, X_{1}, X_{2}, X_{3}\right\}$ to $\mathbb{R}^{4}$ with the basis $\left\{Y_{0}, Y_{1}, Y_{2}, Y_{3}\right\}$, it follows that $A_{n}$ converges to the identity matrix. As a consequence, $g_{n}$ converges to a linear isomorphism $g$ of $\mathbb{R}^{4}$, which must be in $\mathrm{O}(3,1)$ since this subgroup is closed in the linear group. Therefore, $g$ induces an element of $\operatorname{PO}(3,1)$ which, by continuity, sends $P$ to $Q$ as required.

To analyze the local topology of $\widetilde{\mathcal{P}}_{\Gamma}$ and $\mathcal{P}_{\Gamma}$, subdivide the faces of the model projective polyhedron $P_{0}^{\text {Proj }}$ into triangles by adding a few edges (and 
no vertex). Let $\widehat{P}_{0}^{\text {Proj }}$ denote $P_{0}^{\text {Proj }}$ with this new cell decomposition of its boundary. The set $V$ of vertices of $\widehat{P}_{0}^{\text {Proj }}$ is the same as that of $P_{0}^{\text {Proj }}$, however the set of edges of $\widehat{P}_{0}^{\text {Proj }}$ is now $E \cup E^{\prime}$, where $E$ is the set of 'old' edges of $P_{0}^{\text {Proj }}$ and $E^{\prime}$ is the set of 'new' edges introduced to subdivide its faces into triangles.

Let $x \in\left(\mathbb{R} P^{3}\right)^{V}$ be a point which is close to the vertex set $\Phi(P) \in\left(\mathbb{R P}^{3}\right)^{V}$ of a polyhedron $P \in \widetilde{\mathcal{P}}_{\Gamma}$. For a (triangle) face $f$ of $\widehat{P}_{0}^{\text {Proj }}$, with vertices $v_{1}, v_{2}$ and $v_{3}$, the corresponding coordinates $x_{v_{1}}, x_{v_{2}}, x_{v_{3}} \in \mathbb{R P}^{3}$ of $x$ are the vertices of a triangle $t_{f}$ in $\mathbb{R P}^{3}$, uniquely determined if we require that $t_{f}$ is close to the triangle bounded by the corresponding vertices in a face of $P$ (beware that three non-collinear points are the vertices of 4 distinct triangles in $\mathbb{R P}^{3}$ ). As $f$ ranges over all faces of $\widehat{P}_{0}^{\text {Proj }}$, the union of the $t_{f}$ forms the boundary of a polyhedron $P_{x}$ in $\mathbb{R P}^{3}$. This polyhedron $P_{x}$ is a perturbation of $P^{\text {Proj }}$. In contrast to $P^{\text {Proj }}, P_{x}$ is not necessarily convex and some of its vertices may be inside $\mathbb{H}^{3}$. However, for $x$ sufficiently close to $\Phi(P)$, the polyhedron $P_{x}$ is embedded and each of its edges meets $\mathbb{H}^{3}$.

If $e \in E \cup E^{\prime}$ is an edge of $\widehat{P}_{0}^{\text {Proj }}$, consider the corresponding edge of $P_{x}$, and let $\left.\widetilde{\Theta}_{e}(x) \in\right]-\pi, \pi[\subset \mathbb{R}$ be the external dihedral angle of the hyperbolic polyhedron $P_{x} \cap \mathbb{H}^{3}$ along this edge. Considering all such edges $e \in E \cup E^{\prime}$, this defines a map $\widetilde{\Theta}: U \rightarrow \mathbb{R}^{E \cup E^{\prime}}$ on a neighborhood $U$ of $\Phi(P)$ in $\left(\mathbb{R P}^{3}\right)^{V}$. We now use $\widetilde{\Theta}$ to characterize the intersection of $U$ with the image of the embedding $\Phi: \widetilde{\mathcal{P}}_{\Gamma} \rightarrow\left(\mathbb{R} \mathbb{P}^{3}\right)^{V}$.

Lemma 9. - For every $P \in \widetilde{\mathcal{P}}_{\Gamma}$, there is a neighborhood $U$ of $\Phi(P)$ in $\left(\mathbb{R P}^{3}\right)^{V}$ such that, for the function $\widetilde{\Theta}: U \rightarrow \mathbb{R}^{E \cup E^{\prime}}$ defined above, $U \cap \Phi\left(\widetilde{\mathcal{P}}_{\Gamma}\right)$ consists of those $x \in U$ with the following properties:

(i) $\widetilde{\Theta}_{e}(x)=0$ for every ' $n e w$ ' edge $e \in E^{\prime}$;

(ii) for every vertex $v \in V, \sum_{i=1}^{n} \widetilde{\Theta}_{e_{i}}(x) \geq 2 \pi$ where $e_{1}, \ldots, e_{n} \in E$ are the edges of $P_{0}^{\text {Proj }}$ that contain the vertex $v$.

Proof. - Choose $U$ small enough that $\widetilde{\Theta}_{e}(x)>0$ for every 'old' edge $e \in E$ and every $x \in U$. If $x \in U$ satisfies Conditions (i) and (ii), this property of old edges and Condition (i) guarantee that $P_{x}$ is convex and, if we erase those edges where $\partial P_{x}$ is flat, has the same combinatorics as $P_{0}^{\text {Proj }}$. By Lemma 7 , Condition (ii) implies that all the vertices of $P_{x}$ lie outside of $\mathbb{H}^{3}$. Since all the edges of $P_{x}$ already meet $\mathbb{H}^{3}$, this shows that $P_{x} \cap \mathbb{H}^{3}$ is a hyperideal polyhedron, whose image in $\left(\mathbb{R} P^{3}\right)^{V}$ is equal to $x$.

Conversely, Conditions (i) and (ii) are clearly necessary for $x \in U$ to be in $\Phi\left(\widetilde{\mathcal{P}}_{\Gamma}\right)$, by definition of $\widetilde{\Theta}$ and by Proposition 5 .

The key technical step in the proof of the Rigidity Theorem 2 is the following infinitesimal rigidity result, whose proof will occupy the next section.

TOME $130-2002-\mathrm{N}^{\mathrm{O}} 3$ 
Proposition 10 (Infinitesimal Rigidity Lemma). - For $P \in \widetilde{\mathcal{P}}_{\Gamma}$, let $\widetilde{\Theta}$ : $U \rightarrow \mathbb{R}^{E \cup E^{\prime}}$ be the map defined as above on a neighborhood $U$ of $\Phi(P)$ in $\left(\mathbb{R P}^{3}\right)^{V}$. Then, the kernel of the tangent map of $\widetilde{\Theta}$ at $\Phi(P)$ is equal to the image of the tangent map at $\mathrm{Id} \in \mathrm{PO}(3,1)$ of the map $\mathrm{PO}(3,1) \rightarrow\left(\mathbb{R P}^{3}\right)^{V}$ defined by $g \mapsto g \Phi(P)$. In other words, any infinitesimal deformation of $\Phi(P)$ in $\left(\mathbb{R P}^{3}\right)^{V}$ which infinitesimally respects the dihedral angles $\widetilde{\Theta}_{e}$ for all $e \in E \cup E^{\prime}$ must come from composition by an infinitesimal element of $\mathrm{PO}(3,1)$.

Assuming Proposition 10, we are now ready to determine the local type of $\mathcal{P}_{\Gamma}=\widetilde{\mathcal{P}}_{\Gamma} / \mathrm{PO}(3,1)$.

The map $\widetilde{\Theta}$ is defined on a neighborhood of $\Phi\left(\widetilde{\mathcal{P}}_{\Gamma}\right)$, and we can consider the composition $\widetilde{\Theta} \circ \Phi: \widetilde{\mathcal{P}}_{\Gamma} \rightarrow \mathbb{R}^{E \cup E^{\prime}}$. Propositions 5, 6 and Lemma 9 impose constraints on the image of $\widetilde{\Theta} \circ \Phi$. Namely, its image is contained in $K_{\Gamma} \times 0 \subset$ $\mathbb{R}^{E} \times \mathbb{R}^{E^{\prime}}$ where the subset $K_{\Gamma} \subset \mathbb{R}^{E}$ consists of those $\theta \in \mathbb{R}^{E}$ which satisfy the following conditions:

0 ) For every $e \in E$, the coordinate $\theta_{e}$ of $\theta$ corresponding to $e$ is in the interval $] 0, \pi[$

1) For every closed curve $\gamma$ embedded in $\Gamma$ and passing through the edges $e_{1}, e_{2}, \ldots, e_{n}$ of $\Gamma, \sum_{i=1}^{n} \theta_{e_{i}} \geq 2 \pi$ with equality allowed only when $\gamma$ is the boundary of a face of $\mathbb{S}^{2}-\Gamma$.

2) For every arc $\gamma$ embedded in $\Gamma$, passing through the edges $e_{1}, e_{2}, \ldots$, $e_{n}$ of $\Gamma$, and joining two distinct vertices which are in the closure of the same component of $\mathbb{S}^{2}-\Gamma$ but such that $\gamma$ is not contained in the boundary of that component, $\sum_{i=1}^{n} \theta_{e_{i}}>\pi$.

The map $\widetilde{\Theta} \circ \Phi: \widetilde{\mathcal{P}}_{\Gamma} \rightarrow K_{\Gamma} \times 0 \subset \mathbb{R}^{E} \times \mathbb{R}^{E^{\prime}}$ is invariant under the action of $\mathrm{PO}(3,1)$ on $\widetilde{\mathcal{P}}_{\Gamma}$, and therefore induces a map $\Theta: \mathcal{P}_{\Gamma} \rightarrow K_{\Gamma}$ on $\mathcal{P}_{\Gamma}=$ $\widetilde{\mathcal{P}}_{\Gamma} / \mathrm{PO}(3,1)$.

THEOREM 11 (Local characterization of hyperideal polyhedra by their dihedral angles)

The map $\Theta: \mathcal{P}_{\Gamma} \rightarrow K_{\Gamma}$ is a local homeomorphism.

Proof (assuming Proposition 10). - Consider a hyperideal polyhedron $P \in$ $\widetilde{\mathcal{P}}_{\Gamma}$, and its class in $\mathcal{P}_{\Gamma}$ which, to avoid cumbersome notation, we will denote by the same letter $P \in \mathcal{P}_{\Gamma}$. We want to show that $\Theta$ restricts to a homeomorphism from a neighborhood of $P$ in $\mathcal{P}_{\Gamma}$ to a neighborhood of $\Theta(P)$ in $K_{\Gamma}$.

Consider the restriction $\widetilde{\Theta}: U \rightarrow \mathbb{R}^{E \cup E^{\prime}}$ of $\widetilde{\Theta}$ to an open neighborhood $U$ of $\Phi(P)$ in $\left(\mathbb{R}^{3}\right)^{V}$. Because all the faces of the cell decomposition of $\widehat{P}_{0}^{\text {Proj }}$ are triangles, a counting argument shows that $\# E+\# E^{\prime}=3 \# V+6$, where $\# X$ denotes the cardinal of $X$. In particular, the difference between the dimensions of the domain and of the range of $\widetilde{\Theta}: U \rightarrow \mathbb{R}^{E \cup E^{\prime}}$ is equal to 6 . 
The map $\mathrm{PO}(3,1) \rightarrow\left(\mathbb{R P}^{3}\right)^{V}$ defined by $g \mapsto g \Phi(P)$ has an injective tangent map at Id. This can easily be seen by picking, as in the proof of Lemma 8 , four faces $f_{0}, f_{1}, f_{2}, f_{3} \in F$ such that the unit normal vectors $X_{0}, X_{1}, X_{2}$, $X_{2} \in \mathbb{R}^{4}$ of the planes containing the corresponding faces of $P^{\text {Proj }}$ are linearly independent; an element of the kernel of the tangent map infinitesimally fixes the vertices of $P^{\text {Proj }}$, therefore infinitesimally fixes the $X_{i}$, and consequently must be trivial. It follows that the image of the tangent map of $\mathrm{PO}(3,1) \rightarrow$ $\left(\mathbb{R P}^{3}\right)^{V}$ at Id has dimension 6 .

Combining this computation of dimensions with Proposition 10, we conclude that the differential of $\widetilde{\Theta}: U \rightarrow \mathbb{R}^{E \cup E^{\prime}}$ at $\Phi(P)$ is surjective. In particular, $\widetilde{\Theta}(U)$ is open in $\mathbb{R}^{E \cup E^{\prime}}$ if we choose $U$ small enough.

The Submersion Theorem shows that we can choose the neighborhood $U$ of $\Phi(P)$ so that it decomposes as $U \cong U^{\prime} \times U^{\prime \prime}$ in such a way that $\widetilde{\Theta}$ corresponds to the composition of the projection $U^{\prime} \times U^{\prime \prime} \rightarrow U^{\prime}$ and of a diffeomorphism $U^{\prime} \rightarrow \widetilde{\Theta}(U)$. Let $\left(u_{0}^{\prime}, u_{0}^{\prime \prime}\right) \in U^{\prime} \times U^{\prime \prime}$ correspond to $\Phi(P) \in U$. For every $u^{\prime} \in U^{\prime}$, the map $g \mapsto g\left(u^{\prime}, u_{0}^{\prime \prime}\right)$ immerses a neighborhood $W_{u^{\prime}}$ of Id in $\mathrm{PO}(3,1)$ in the slice $\left\{u^{\prime}\right\} \times U^{\prime \prime} \subset U$ since $\widetilde{\Theta} \circ g=\widetilde{\Theta}$. By the above dimension computations, $\mathrm{PO}(3,1)$ and $U^{\prime \prime}$ both have dimension 6 , and we conclude that we can choose the neighborhood $U$ and the identification $U \cong U^{\prime} \times U^{\prime \prime}$ so that each slice $\left\{u^{\prime}\right\} \times U^{\prime \prime}$ is of the form $W\left(u^{\prime}, u_{0}^{\prime \prime}\right)$, where $W$ is a fixed neighborhood of $\operatorname{Id}$ in $\mathrm{PO}(3,1)$.

We claim that, if $U$ and $W$ are chosen small enough, two elements of $U$ will be in the same $\operatorname{PO}(3,1)$-orbit if and only if they belong to the same slice $\left\{u^{\prime}\right\} \times U^{\prime \prime} \subset U$. It clearly suffices to show that, if $x \in\left(\mathbb{R P}^{3}\right)^{V}$ and $g \in \operatorname{PO}(3,1)$ are such that $x$ and $g x$ are both close to $\Phi(P)$, then $g$ must be close to Id in $\mathrm{PO}(3,1)$. For this, pick again four faces $f_{0}, f_{1}, f_{2}, f_{3} \in F$ such that the unit normal vectors $X_{0}, X_{1}, X_{2}, X_{2} \in \mathbb{R}^{4}$ of the planes containing the corresponding faces of $P^{\text {Proj }}$ are linearly independent. Then, as in the proof of Lemma 8, each $g X_{i}$ is close to $X_{i}$, and it follows that $g$ is close to $\operatorname{Id}$ in $\operatorname{PO}(3,1)$.

Therefore, if we choose $U$ small enough, two points $\left(u^{\prime}, u^{\prime \prime}\right),\left(v^{\prime}, v^{\prime \prime}\right) \in U^{\prime} \times$ $U^{\prime \prime} \cong U$ are in the same $\mathrm{PO}(3,1)$-orbit if and only if they are in the same slice $\left\{u^{\prime}\right\} \times U^{\prime \prime}$, namely if and only if $v^{\prime}=u^{\prime}$, namely if and only if they have the same image under $\widetilde{\Theta}$. In other words, the restriction of $\widetilde{\Theta}$ to $U$ induces a homeomorphism between $U / \mathrm{PO}(3,1)$ and $\widetilde{\Theta}(U)$.

By Lemma 9, the image of $U \cap \Phi\left(\widetilde{\mathcal{P}}_{\Gamma}\right)$ is equal to $\widetilde{\Theta}(U) \cap\left(K_{\Gamma} \times\{0\}\right)$. It follows that $\widetilde{\Theta}$ induces a homeomorphism between $U \cap \Phi\left(\widetilde{\mathcal{P}}_{\Gamma}\right) / \mathrm{PO}(3,1)$ and $\widetilde{\Theta}(U) \cap\left(K_{\Gamma} \times\{0\}\right)$. Since $\Phi$ is a $\mathrm{PO}(3,1)$-equivariant homeomorphism between $\widetilde{\mathcal{P}}_{\Gamma}$ and $\Phi\left(\widetilde{\mathcal{P}}_{\Gamma}\right)$, we conclude that $\widetilde{\Theta} \circ \Phi$ induces a homeomorphism between $\Phi^{-1}(U) / \mathrm{PO}(3,1)$ and $\widetilde{\Theta}(U) \cap\left(K_{\Gamma} \times\{0\}\right)$. Note that $\Phi^{-1}(U) / \mathrm{PO}(3,1)$ is an open neighborhood of $P$ in $\mathcal{P}_{\Gamma}$, and that $\widetilde{\Theta}(U) \cap\left(K_{\Gamma} \times\{0\}\right)$ is an open neighborhood of $\widetilde{\Theta} \circ \Phi(P)$ in $K_{\Gamma} \times\{0\}$ since $\widetilde{\Theta}(U)$ is open in $\mathbb{R}^{E} \times \mathbb{R}^{E^{\prime}}$. 
This proves that the map $\Theta: \mathcal{P}_{\Gamma} \rightarrow K_{\Gamma}$ restricts to a homeomorphism from a neighborhood of $P$ in $\mathcal{P}_{\Gamma}$ to a neighborhood of $\Theta(P)$ in $K_{\Gamma}$.

\section{The Infinitesimal Rigidity Lemma}

This section is devoted to the proof of the Infinitesimal Rigidity Lemma of Proposition 10. The proof follows the general lines of the famous arguments of Cauchy [5] on the deformations of convex polyhedra in $\mathbb{E}^{3}$ (see also [15][7]), as adapted to hyperbolic polyhedra by Andreev [2] and Rivin [12]. For polyhedra which are strictly hyperideal, namely whose vertices are all outside of the closure of $\mathbb{H}^{3}$, this proof is essentially contained in $[12, \S 4]$ if we use the truncated polyhedron introduced in $\S 1$.

Recall that we have a map $\widetilde{\Theta}: U \rightarrow \mathbb{R}^{E \cup E^{\prime}}$, defined on a neighborhood $U$ of $\Phi(P)$ in $\left(\mathbb{R P}^{3}\right)^{V}$, which to $x \in U$ associates the hyperbolic external dihedral angles of the polyhedron $P_{x}$ whose vertices are the coordinates of $x$ and whose combinatorial type is that of $\widehat{P}_{0}^{\text {Proj }}$. We also have a map $G: \operatorname{PO}(3,1) \rightarrow\left(\mathbb{R P}^{3}\right)^{V}$ defined by $g \mapsto g \Phi(P)$. We want to show that the kernel of the tangent map $T_{\Phi(P)} \widetilde{\Theta}: T_{\Phi(P)}\left(\mathbb{R P}^{3}\right)^{V} \rightarrow \mathbb{R}^{E \cup E^{\prime}}$ is equal to the image of

$$
T_{\mathrm{Id}} G: T_{\mathrm{Id}} \mathrm{PO}(3,1) \longrightarrow T_{\Phi(P)}\left(\mathbb{R P}^{3}\right)^{V} \text {. }
$$

Let $\dot{x} \in T_{\Phi(P)}\left(\mathbb{R P}^{3}\right)^{V}$ be a vector which is contained in the kernel of $T_{\Phi(P)} \widetilde{\Theta}$. We want to show that $\dot{x}$ is in the image of $T_{\mathrm{Id}} G$, namely is tangent to an infinitesimal deformation of $\Phi(P)$ by an infinitesimal element of $\operatorname{PO}(3,1)$.

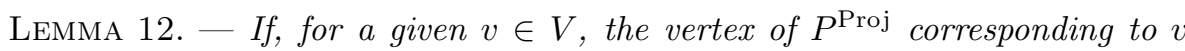
is located on the sphere at infinity $\partial_{\infty} \mathbb{H}^{3} \subset \mathbb{R P}^{3}$, then the coordinate vector $\dot{x}_{v}$ of $\dot{x}$ corresponding to $v$ is tangent to $\partial_{\infty} \mathbb{H}^{3}$ in $\mathbb{R} \mathbb{P}^{3}$.

Proof. - Let $e_{1}, e_{2}, \ldots, e_{n} \in E \cup E^{\prime}$ be the edges of $\widehat{P}_{0}^{\text {Proj }}$ that are adjacent to the vertex $v$. Let $\varphi: U \rightarrow \mathbb{R}$ be the function defined by $\varphi(x)=\sum_{i=1}^{n} \widetilde{\Theta}_{e_{i}}(x)$. Note that the vector $\dot{x}$ is contained in the kernel of the tangent map $T_{\Phi(P)} \varphi$, since it is contained in the kernel of $T_{\Phi(P)} \widetilde{\Theta}$.

By Proposition 5 and Lemma 7, $\varphi(x)=2 \pi$ whenever the coordinate $x_{v}$ of $x \in U \subset\left(\mathbb{R P}^{3}\right)^{V}$ corresponding to $v$ belongs to $\partial_{\infty} \mathbb{H}^{3}$. It follows that the kernel of $T_{\Phi(P)} \varphi$ contains all those vectors $\dot{y} \in T_{\Phi(P)}\left(\mathbb{R P}^{3}\right)^{V}$ whose coordinate $\dot{y}_{v}$ is tangent to $\partial_{\infty} \mathbb{H}^{3}$. The lemma will be proved if we show that the kernel of $T_{\Phi(P)} \varphi$ consists only of these $\dot{y}$ with $\dot{y}_{v}$ tangent to $\partial_{\infty} \mathbb{H}^{3}$. For this, it suffices to show that $T_{\Phi(P)} \varphi$ is non-trivial.

Modifying $P$ by an element of $\mathrm{PO}(3,1)$ if necessary, we can arrange that the

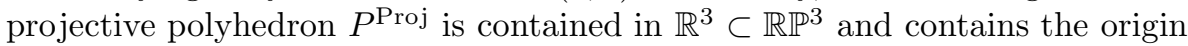
$0 \in \mathbb{H}^{3} \subset \mathbb{R}^{3}$ in its interior.

Let $\left.t \mapsto x^{t} \in U \subset\left(\mathbb{R P}^{3}\right)^{V}, t \in\right]-\varepsilon, \varepsilon\left[\right.$, be a curve such that $x^{0}=\Phi(P)$, such that the coordinate $x_{v^{\prime}}^{t}$ is equal to $x_{v^{\prime}}^{0}$ for every $v^{\prime} \neq v$ and every $t$, and 
such that the curve $t \mapsto x_{v}^{t} \in \mathbb{R P}^{3}$ is tangent to the outer unit normal vector of $\partial_{\infty} \mathbb{H}^{3} \subset \mathbb{R}^{3} \subset \mathbb{R P}^{3}$ at $t=0$. If $\dot{x}^{0}$ denotes the tangent vector of $t \mapsto x^{t}$ at $t=0$, we will use a little bit of euclidean geometry to estimate $T_{\Phi(P)} \varphi\left(\dot{x}^{0}\right)=$ $\mathrm{d} \varphi\left(x^{t}\right) / \mathrm{d} t_{\mid t=0}$.

Consider the projective polyhedron $P_{x^{t}}$ associated to $x^{t} \in\left(\mathbb{R P}^{3}\right)^{V}$, namely with vertex set $x^{t}$ and with the combinatorial type of $\widehat{P}_{0}^{\text {Proj }}$. For $t \geq 0$, the intersection of $P_{x^{t}}$ with $\partial_{\infty} \mathbb{H}^{3}$ has one component $A_{t}$ which is near $x_{v}^{0} \in \partial_{\infty} \mathbb{H}^{3}$. This component $A_{t}$ is a polygon on the sphere $\partial_{\infty} \mathbb{H}^{3}$, reduced to a point when $t=0$. Remember that, if $\Pi$ and $\Pi^{\prime}$ are two projective planes which meet $\mathbb{H}^{3}$, the hyperbolic dihedral angle between the hyperbolic planes $\Pi \cap \mathbb{H}^{3}$ and $\Pi^{\prime} \cap \mathbb{H}^{3}$ is equal to the euclidean angle between the two circles $\Pi \cap \partial_{\infty} \mathbb{H}^{3}$ and $\Pi^{\prime} \cap \partial_{\infty} \mathbb{H}^{3}$, for the euclidean metric on the sphere $\partial_{\infty} \mathbb{H}^{3} \subset \mathbb{R}^{3}$. Applying the Gauss-Bonnet formula to $A_{t}$, we conclude that

$$
\varphi\left(x^{t}\right)=\sum_{i=1}^{n} \widetilde{\Theta}_{e_{i}}\left(x^{t}\right)=2 \pi-\int_{\partial A_{t}} \kappa-\operatorname{area}\left(A_{t}\right)
$$

where $\kappa$ is the geodesic curvature of the boundary $\partial A_{t}$ in $\partial_{\infty} \mathbb{H}^{3}$.

Let us differentiate in $t$ at $t=0$. The area term is bounded by a quantity of order 2 in $t$, and therefore does not contribute to the derivative. Because we arranged that the point 0 is in the interior of $P$, the curvature $\kappa$ is negative bounded away from 0 on each side of $A_{t}$. On the other hand, because the component $\dot{x}_{v}^{0}$ of the tangent vector $\dot{x}^{0}$ is equal to the outer unit normal vector of $\partial_{\infty} \mathbb{H}^{3}$, the length of $\partial A_{t}$ has a positive derivative with respect to $t$ at $t=0$. It follows that $T_{\Phi(P)} \varphi\left(\dot{x}^{0}\right)=\mathrm{d} \varphi\left(x^{t}\right) / \mathrm{d} t_{\mid t=0}$ is strictly positive. (Note that we do not need to worry about the derivative of $\kappa$ since $\partial A_{0}$ has length 0 .)

This proves that $T_{(p, x)} \varphi$ is non-trivial. As a consequence, its kernel consists of those vectors $\dot{y} \in T_{\Phi(P)}\left(\mathbb{R P}^{3}\right)^{V}$ whose coordinate $\dot{y}_{v}$ is tangent to $\partial_{\infty} \mathbb{H}^{3}$. Since $\dot{x}$ belongs to this kernel, this completes the proof of Lemma 12.

Let $\left.t \mapsto x^{t}, t \in\right]-\varepsilon, \varepsilon\left[\right.$, be a smooth curve in $\left(\mathbb{R P}^{3}\right)^{V}$ such that $x^{0}=\Phi(P)$ and $\mathrm{d} x^{t} / \mathrm{d} t_{t=0}=\dot{x}$. By Lemma 12 , we can choose this curve so that, whenever the coordinate $x_{v}^{0}$ of $x^{0}=\Phi(P)$ is in the sphere $\partial_{\infty} \mathbb{H}^{3}$, the corresponding coordinate $x_{v}^{t}$ of $x^{t}$ remains in $\partial_{\infty} \mathbb{H}^{3}$ for every $t$. In particular, for $t$ sufficiently small, all coordinates $x_{v}^{t}$ of $x^{t}$ are in the complement of $\mathbb{H}^{3}$ in $\mathbb{R P}^{3}$.

Again, let $P_{x^{t}}$ be the projective polyhedron associated to $x^{t} \in\left(\mathbb{R P}^{3}\right)^{V}$, namely with vertex set $x^{t}$ and with the combinatorial type of $\widehat{P}_{0}^{\text {Proj }}$.

As in $\S 1$, consider the truncated polyhedron $P_{x^{t}}^{\text {Trun }}$ obtained from $P_{x^{t}}$ by chopping off its intersection with the interiors of disjoint half-spaces and horoballs $H_{v}^{t}$ where, for each $v \in V, H_{v}^{t}$ is a half-space delimited by the hyperbolic plane $\mathbb{H}^{3} \cap\left(x_{v}^{t}\right)^{\perp}$ if the vertex $x_{v}^{t}$ of $P_{x^{t}}$ is strictly hyperideal and $H_{v}^{t}$ is a horoball centered at $x_{v}^{t}$ if this vertex is ideal. From its construction, $P_{x^{t}}^{\text {Trun }}$ inherits a natural polyhedron structure where its faces are of three types:

TOME $130-2002-\mathrm{N}^{\mathrm{O}} 3$ 
(i) the intersection of a face of $P_{x^{t}}$ with the complement in $\mathbb{H}^{3}$ of the interiors of all the $H_{v}^{t}$; such a face is a polygon contained in a hyperbolic plane, whose sides are either geodesic or horocyclic, and whose internal angles are all equal to $\frac{1}{2} \pi$.

(ii) the intersection of $P_{x^{t}}$ with the hyperbolic plane $\partial H_{v}^{t}$ associated to a strictly hyperideal vertex $x_{v}^{t}$; such a face is a hyperbolic polygon with geodesic sides and, at each of its vertices, its internal angle is equal to the dihedral angle of the corresponding edge of $P_{x^{t}} \cap \mathbb{H}^{3}$.

(iii) the intersection of $P_{x^{t}}$ with the horosphere $\partial H_{v}^{t}$ associated to an ideal vertex $x_{v}^{t}$; such a face is a euclidean polygon with geodesic sides and, at each of its vertices, its internal angle is equal to the internal dihedral angle of the corresponding edge of $P_{x^{t}} \cap \mathbb{H}^{3}$.

Recall that in the construction of $P_{x^{t}}^{\text {Trun }}$ we have a degree of freedom in the choice of the horospherical $H_{v}^{t}$ associated to the ideal vertices of $P_{x^{t}}$. We will use this freedom to impose an additional condition on the faces of type (iii). By adjusting the height of the corresponding horospheres $\partial H_{v}^{t}$, we can always arrange that the area of each face of type (iii) is constant, independent of $t$.

Note that the polyhedra $P_{x^{t}}^{\text {Trun }}$ all have the combinatorial type of the polyhedron $\widehat{P}_{0}^{\text {Trun }}$ obtained by truncating the polyhedron $\widehat{P}_{0}^{\text {Proj }}$ (which, as a reminder, was defined by subdividing the faces of $P_{0}^{\text {Proj }}$ into triangles).

Consider now the unsubdivided polyhedron $P_{0}^{\text {Proj }}$, and truncate it to $P_{0}^{\text {Trun }}$. Each vertex $v$ of $P_{0}^{\text {Trun }}$ is also a vertex of $\widehat{P}_{0}^{\text {Trun }}$, and is therefore associated to a vertex $v^{t}$ of $P_{x^{t}}^{\text {Trun }}$.

For each edge $e$ of $P_{0}^{\text {Trun }}$, look at its end vertices $v_{1}, v_{2}$, and consider the hyperbolic distance $d\left(v_{1}^{t}, v_{2}^{t}\right)$ between the corresponding vertices $v_{1}^{t}, v_{2}^{t}$ of $P_{x^{t}}^{\text {Trun }}$. Label the edge $e$ by the symbol,+ 0 or - according to whether the derivative of $d\left(v_{1}^{t}, v_{2}^{t}\right)$ at $t=0$ is positive, zero or negative.

Our goal is to show that all the edges are actually labelled by 0 . This will require a few preparatory lemmas.

If $Q$ is a polygon with each of its edges labelled by a symbol,+ 0 or - , define the number of sign changes of $\partial Q$ as the minimum number of vertices $v_{1}, \ldots, v_{n}$ which one needs to remove from $\partial Q$ so that the edges in each component of $\partial Q-\left\{v_{1}, \ldots, v_{n}\right\}$ have the same sign, namely are either all in $\{+, 0\}$ or all in $\{-, 0\}$. Note that the number of sign changes is always even.

We will first show that, for each face $f$ of $P_{0}^{\text {Trun }}$, either all the edges of $f$ are labelled by 0 , or it admits at least 4 sign changes in its boundary. In other words, there cannot be 0 or 2 sign changes in the boundary of $f$, unless all the edges of $f$ are labelled by 0 .

We begin with faces of Type (iii), contained in horospheres. The argument uses the following euclidean geometry lemma, which is an infinitesimal and simpler version of Lemma $\mathrm{M}_{3}$ of [15] (a result attributed there to A.D. Aleksandrov). 
LEMMA 13. - In the euclidean plane $\mathbb{R}^{2}$, let $\left.Q_{t}, t \in\right]-\varepsilon, \varepsilon[$, be a differentiable family of strictly convex polygons with straight sides. Suppose that, at $t=0$, the derivative of the angle of $Q_{t}$ at each of its vertices is equal to 0 , and that the derivative of the area of $Q_{t}$ is also equal to 0 . Label each edge of $Q_{0}$ by the symbol,+ 0 , or - according to whether the derivative of its length at $t=0$ is positive, zero or negative. Then, either all edges are labelled by 0 , or there are at least 4 sign changes in $\partial Q_{0}$.

Here, "strictly convex" means that the internal angle of $Q_{t}$ at each of its vertices is strictly between 0 and $\pi$.

Proof. - (Compare [15, Lemma $\left.\left.\mathrm{M}_{3}\right].\right)$ Suppose, in search of a contradiction, that there are exactly 2 sign changes. We can then index the edges of $Q_{t}$ as $e_{1}^{t}, \ldots, e_{q}^{t}, e_{q+1}^{t}, \ldots, e_{r}^{t}, e_{r+1}^{t}=e_{1}^{t}$, going counterclockwise in this order around $\partial Q_{t}$, in such a way that $e_{i}^{t}$ is labelled by + or 0 if $1 \leq i \leq q$, and by - or 0 if $q+1 \leq i \leq r$. Let $v_{i}^{t}$ be the vertex intersection of $e_{i}^{t}$ and $e_{i+1}^{t}$, and set $v_{0}^{t}=v_{r}^{t}$ for consistency of the notation. Finally, let $T_{i}^{t}$ be the unit tangent vector of the edge $e_{i}^{t}$, oriented by the counterclockwise orientation of $\partial Q_{t}$, and let $\ell_{i}^{t}$ be the length of $e_{i}^{t}$. Then, for $1 \leq i<j \leq r$,

$$
v_{j}^{t}-v_{i}^{t}=\sum_{k=i+1}^{j} \ell_{k}^{t} T_{k}^{t}=-\sum_{k=1}^{i} \ell_{k}^{t} T_{k}^{t}-\sum_{k=j+1}^{r} \ell_{k}^{t} T_{k}^{t} .
$$

Let $\left.\alpha_{i}^{t} \in\right] 0, \pi\left[\right.$ be the angle from $T_{i}^{t}$ to $T_{i+1}^{t}$, namely the external angle of $Q_{t}$ at the vertex $v_{i}^{t}$. Note that $\sum_{i=1}^{r} \alpha_{i}^{0}=2 \pi$. Replacing $t$ by $-t$ if necessary (which exchanges the labels + and - ), we can assume without loss of generality that $\sum_{i=q+1}^{r} \alpha_{i}^{0} \leq \pi$. Pick an index $p$ with $1 \leq p \leq q$ such that $\sum_{i=1}^{p-1} \alpha_{i}^{0}<\pi$ and $\sum_{i=p+1}^{q} \alpha_{i}^{0}<\pi$.

Composing $Q_{t}$ by an isometry depending differentiably on $t$, we can arrange that the vertex $v_{p}^{t}$ is fixed, and that the tangent vector $T_{p}^{t}$ is constant. By our hypothesis that the angles of $Q_{t}$ have derivative 0 at $t=0$, it follows that $\mathrm{d} T_{i}^{t} / \mathrm{d} t_{\mid t=0}=0$ for every $i$. As a consequence, if $\dot{\ell}_{i}^{0}=\mathrm{d} \ell_{i}^{t} / \mathrm{d} t_{\mid t=0}$ denotes the derivative of the length $\ell_{i}^{t}$ at $t=0$, the vector $\dot{v}_{i}^{0}=\mathrm{d} v_{i}^{t} / \mathrm{d} t_{\mid t=0}$ is equal to

$$
\dot{v}_{i}^{0}= \begin{cases}+\sum_{j=p+1}^{i} \dot{\ell}_{j}^{0} T_{j}^{0} & \text { if } p+1 \leq i \leq r, \\ -\sum_{j=i+1}^{p} \dot{\ell}_{j}^{0} T_{j}^{0} & \text { if } 1 \leq i \leq p-1 .\end{cases}
$$

We will consider the direction in which the edges $e_{i}^{t}$ move. We will say that $e_{i}^{t}$ weakly moves towards (resp. away from) $Q_{0}$ if either the vector $\dot{v}_{i-1}^{0}=$ $\mathrm{d} v_{i-1}^{t} / \mathrm{d} t_{\mid t=0}$ is equal to 0 or if the angle from $\dot{v}_{i-1}^{0}$ to $T_{i}^{0}$ is in the closed interval $[-\pi, 0]$ (resp. $[0, \pi]$ ); note that we can consider the vector $\dot{v}_{i}^{0}=\dot{v}_{i-1}^{0}+\dot{\ell}_{i}^{0} T_{i}^{0}$ instead of $\dot{v}_{i-1}^{0}$ for this property. The edge $e_{i}^{t}$ strictly moves towards (resp. away from) $Q_{0}$ if $\dot{v}_{i-1}^{0}=\mathrm{d} v_{i-1}^{t} / \mathrm{d} t_{\mid t=0}$ is non-zero and the angle from $\dot{v}_{i-1}^{0}$ to $T_{i}^{0}$ is in the open interval $]-\pi, 0[$ (resp. $] 0, \pi[$ ).

TOME $130-2002-\mathrm{N}^{\mathrm{O}} 3$ 
For $p+1 \leq j \leq i-1 \leq q$, the derivative $\dot{\ell}_{j}^{0}$ is non-negative and the angle from $T_{j}^{0}$ to $T_{i}^{0}$ is in the interval $] 0, \pi[$, by choice of the indexing. It follows that the angle from $\dot{v}_{i-1}^{0}=\sum_{j=p+1}^{i-1} \dot{\ell}_{j}^{0} T_{j}^{0}$ to $T_{i}^{0}$ is in $[0, \pi]$, unless $\dot{v}_{i-1}^{0}=0$. In other words, every edge $e_{i}^{t}$ with $p+2 \leq i \leq q+1$ weakly moves away from $Q_{0}$. In addition, $e_{i}^{t}$ strictly moves away from $Q_{0}$ if there is a $j$ with $p+1 \leq j \leq i-1 \leq q-1$ and $\dot{\ell}_{j}^{0}>0$, namely such that $e_{j}^{0}$ is labelled by the symbol + .

For $1 \leq i \leq p-1$, the same argument applied to $\dot{v}_{i}^{0}=-\sum_{j=i+1}^{p} \dot{\ell}_{j}^{0} T_{j}^{0}$ shows that $e_{i}^{t}$ moves away from $Q_{0}$, and does so strictly if there is a $j$ with $i+1 \leq j \leq p$ such that $e_{j}^{0}$ is labelled by the symbol + .

In particular, for $1 \leq i \leq q+1$, the edge $e_{i}^{t}$ moves away from $Q_{0}$. In addition, since there is at least one edge labelled by the symbol + , at least one of the two edges $e_{1}^{t}$ and $e_{q+1}^{t}$ strictly moves away from $Q_{0}$. Since the areas of $Q_{t}$ is infinitesimally constant, we conclude that there must be an index $i_{0}$, with $q+2 \leq i_{0} \leq r$, such that $e_{i_{0}}^{t}$ strictly moves towards of $Q_{0}$. We can choose this $i_{0}$ so that $e_{i_{0}+1}^{t}$ moves away from $Q_{0}$ (using the convention that $e_{r+1}^{t}=e_{1}^{t}$ when $\left.i_{0}=r\right)$.

Because $e_{i_{0}}^{t}$ strictly moves towards $Q_{0}$ and $e_{i_{0}+1}^{t}$ moves away from $Q_{0}$, the vector $\dot{v}_{i_{0}}^{0}$ is in the angular sector from $T_{i_{0}}^{0}$ to $T_{i_{0}+1}^{0}$. Since $\sum_{i=q+1}^{r} \alpha_{i}^{0} \leq \pi$, we conclude that the angle from $\dot{v}_{i_{0}}^{0}$ to $T_{q+1}$ is in $]-\pi, 0[$. Remembering that $\dot{\ell}_{j}^{0} \leq 0$ for all $j$ with $q+1 \leq j \leq r$, we conclude that the angle from $\dot{v}_{q+1}^{0}=\dot{v}_{i_{0}}^{0}-\sum_{j=q+2}^{i_{0}} \dot{\ell}_{j}^{0} T_{j}^{0}$ to $T_{q+1}$ is in $]-\pi, 0[$. However, this contradicts our earlier conclusion that $e_{q+1}^{t}$ moves away from $Q_{0}$.

This contradiction shows that there cannot be 2 sign changes around $Q_{0}$. A similar but simpler argument shows that there cannot be 0 sign change, unless all edges are labelled by 0 . Therefore, either all edges are labelled by 0 , or there must be at least 4 sign changes around $Q_{0}$.

LEMma 14. - For every face $Q$ of type (iii) of the truncated polyhedron $P_{0}^{\text {Trun }}$, either all the edges of $\partial Q$ are labelled by 0 , or there are at least 4 sign changes in $\partial Q$.

Proof. - There is a face $\widehat{Q}_{t}$ of the truncated polyhedron $P_{x^{t}}^{\text {Trun }}$ which is associated to $Q$ and is contained in a horosphere $\partial H_{t}$. Note that the vertices of $\widehat{Q}_{t}$ correspond to the edges of $P_{x^{t}}$ that are adjacent to the vertex of $P_{x^{t}}$ facing $\widehat{Q}_{t}$. In particular, some vertices of $\widehat{Q}_{t}$ are naturally associated to vertices of $Q$ and other vertices are not. In the horosphere $\partial H_{t}$ endowed with its euclidean metric, let $Q_{t}$ be the convex hull of those vertices of $\widehat{Q}_{t}$ which correspond to vertices of $Q$.

For an edge $e$ of $Q$, with end vertices $v_{1}$ and $v_{2}$, the hyperbolic distance $d\left(v_{1}^{t}, v_{2}^{t}\right)$ between the corresponding vertices $v_{1}^{t}, v_{2}^{t}$ of $P_{x^{t}}^{\text {Trun }}$ in $\mathbb{H}^{3}$ is a strictly 
increasing function of the length of the edge $e_{t}$ of $Q_{t}$ corresponding to $e$. It follows that, in our labelling of the edges of $P_{0}^{\text {Trun }}$, the edge $e$ is labelled by,+ 0 , or - exactly when the derivative of the length of $e_{t}$ at $t=0$ is positive, zero or negative, respectively.

Because the vector $\dot{x} \in T_{\Phi(P)}\left(\mathbb{R P}^{3}\right)^{V}$ tangent to $t \mapsto x_{t}$ is contained in the kernel of the tangent map $T_{\Phi(P)} \widetilde{\Theta}$, all the angles of $\widehat{Q}_{t}$ have derivative 0 at $t=0$. In particular, because $\widehat{Q}_{0}=Q_{0}$, the distance from each vertex of $\widehat{Q}_{t}$ to $\partial Q_{t}$ has derivative 0 at $t=0$. This provides two important conclusions: First, the angles of $Q_{t}$ have derivative 0 at $t=0$; second, the area of $Q_{t}$ has the same derivative as the area of $\widehat{Q}_{t}$ at $t=0$, namely 0 since the area of $\widehat{Q}_{t}$ is constant by choice of the truncated polyhedron $P_{x^{t}}^{\text {Trun }}$.

We can therefore apply Lemma 13 to the polyhedron $Q_{t}$, which provides the conclusion of Lemma 14.

For faces of Type (i) of $P_{0}^{\text {Trun }}$, we use the following lemma.

LEMma 15. - In the hyperbolic plane $\mathbb{H}^{2}$, let $\left.Q_{t}, t \in\right]-\varepsilon, \varepsilon[$, be a differentiable family of convex hyperideal polygons such that, for every vertex $v$ of $Q_{t}^{\text {Proj }}$ which is on the circle at infinity $\partial_{\infty} \mathbb{H}^{2}$, the corresponding vertex $v_{t}$ is also on $\partial_{\infty} \mathbb{H}^{2}$ for every $t$. Let $Q_{t}^{\text {Trun }}$ be obtained by truncating $Q_{t}$, chosen to depend differentiably on $t$ (remember that the truncation is not uniquely determined at the ideal vertices of $\left.Q_{t}\right)$. Label each edge of $Q_{0}^{\text {Trun }}$ by the symbol,+ 0 , or according to whether the derivative of its length at $t=0$ is positive, zero or negative. Then, either all edges are labelled by 0 , or there are at least 4 sign changes in $Q_{0}^{\text {Trun }}$.

Proof. - If there are fewer than 4 sign changes, we can then index the edges of $Q_{t}^{\text {Trun }}$ as $e_{1}^{t}, \ldots, e_{p}^{t}, e_{p+1}^{t}, \ldots, e_{q}^{t}, e_{q+1}^{t}=e_{1}^{t}$, going counterclockwise in this order around $\partial Q_{t}^{\text {Trun }}$, in such a way that $e_{i}^{0}$ is labelled by + or 0 if $2 \leq i \leq p-1$, and by - or 0 if $p+1 \leq i \leq q$. In addition, replacing them by suitable neighbors if necessary, we can arrange that $e_{1}^{t}$ and $e_{p}^{t}$ are both contained in edges of the original untruncated polyhedron $Q_{t}$.

We use the projective model for the hyperbolic plane $\mathbb{H}^{2} \subset \mathbb{R}^{2} \subset \mathbb{R P}^{2}$, with isometry group $\mathrm{PO}(2,1)$. For each $i$, let $T_{i}^{u} \in \mathrm{PO}(2,1)$ be the hyperbolic isometry which acts by translation of $u \in \mathbb{R}$ along the geodesic or horocycle that contains $e_{i}^{0}$, for the orientation coming from the counterclockwise orientation of $\partial Q_{0}^{\text {Trun }}$. Let $g_{i}^{t}$ be the geodesic or horocycle of $\mathbb{H}^{2}$ that contains the edge $e_{i}^{t}$, transversely oriented by the outer normal orientation of $\partial Q_{0}^{\text {Trun }}$. Finally, let $N_{1}^{t}$ and $N_{p}^{t} \in \mathbb{R}^{3}$ be the unit normal vectors of the transversely oriented geodesics $g_{1}^{t}$ and $g_{p}^{t}$, as defined (for the 3 -dimensional case) in $\S 1$.

Composing $Q_{t}$ by an isometry depending differentiably on $t$ if necessary, we can arrange that $e_{1}^{t}$ stays in a fixed geodesic of $\mathbb{H}^{2}$, and that the vertex separating $e_{1}^{t}$ from $e_{2}^{t}$ is constant. Since all the angles of $Q_{t}^{\text {Trun }}$ remain equal to $\frac{1}{2} \pi$,

TOME $130-2002-\mathrm{N}^{\mathrm{O}} 3$ 
we conclude that $N_{p}^{t}=T_{2}^{\ell_{2}^{t}-\ell_{2}^{0}} T_{3}^{\ell_{3}^{t}-\ell_{3}^{0}} \ldots T_{p-1}^{\ell_{p-1}^{t}-\ell_{p-1}^{0}} N_{p}^{0}$ where $\ell_{i}^{t}$ denotes the length of $e_{i}^{t}$. Using dots to demote derivatives with respect to $t$, as usual, this gives $\dot{N}_{p}^{0}=\sum_{i=2}^{p-1} \dot{\ell}_{i}^{0} \dot{T}_{i}^{0} N_{p}^{0}$ for the infinitesimal isometry $\dot{T}_{i}^{0}=\mathrm{d} T_{i}^{u} / \mathrm{d} u_{\mid t=0} \in$ $T_{\mathrm{Id}} \mathrm{PO}(3,1)$.

We claim that $B\left(N_{1}^{0}, \dot{T}_{i}^{0} N_{p}^{0}\right) \geq 0$, and that the inequality is strict in all but one case.

For this, first consider the case where $e_{i}^{0}$ is geodesic. Composing by an isometry of $\mathbb{H}^{2}$, we can arrange that $g_{i}^{0}$ is equal to the geodesic going from $(-1,0)$ to $(+1,0)$ in $\mathbb{H}^{2} \subset \mathbb{R}^{2} \subset \mathbb{R P}^{2}$, and that $Q_{0}^{\text {Trun }}$ is locally above this geodesic. We still have a degree of freedom through hyperbolic translations along this geodesic. Composing with such a translation, we can arrange that the point $(0,0)$ is located on $g_{i}^{0}$ between the point which is closest to $g_{1}^{0}$ and the point which is closest to $g_{p}^{0}$. Then, because $e_{1}^{0}, e_{i}^{0}$ and $e_{p}^{0}$ arise in this order for the counterclockwise orientation of $\partial Q_{0}^{\text {Trun }}$, the vector $N_{1}^{0}=\left(a_{1}, b_{1}, c_{1}\right)$ points to the left of $(0,0)$ and $N_{p}^{0}=\left(a_{p}, b_{p}, c_{p}\right)$ points to the right in the sense that $a_{1}>0, b_{1}<0, a_{p}>0, b_{p}>0$. (There are several cases to consider, according to whether $g_{1}^{0}$ and $g_{p}^{0}$ meet $g_{i}^{0}$ or not). An easy computation now gives that $\dot{T}_{i}^{0}$ is given by the matrix $\left(\begin{array}{lll}0 & 1 & 0 \\ 1 & 0 & 0 \\ 0 & 0 & 0\end{array}\right)$ so that $B\left(N_{1}^{0}, \dot{T}_{i}^{0} N_{p}^{0}\right)=a_{1} b_{p}-a_{p} b_{1}>0$.

Similarly, when $e_{i}^{0}$ is horocyclic, we can arrange by an isometry of $\mathbb{H}^{2}$ that the horocyle $g_{i}^{0}$ is centered at the point $(-1,0)$, namely touches $\partial_{\infty} \mathbb{H}^{2}$ at that point. We now have to distinguish subcases.

If the geodesics $g_{1}^{0}$ and $g_{p}^{0}$ are not asymptotic, we can arrange that the point $(0,0)$ is located in the interior of the shortest geodesic arc joining $g_{1}^{0}$ to $g_{p}^{0}$. Then $N_{1}^{0}=\left(a_{1}, b_{1}, c_{1}\right)$ and $N_{p}^{0}=\left(a_{p}, b_{p}, c_{p}\right)$ are such that $a_{1}>0, b_{1}=0$, $c_{1}>0, a_{p}>0, b_{p}=0$ and $c_{p}<0$. Also, $\dot{T}_{i}^{0}$ is given by the matrix $\left(\begin{array}{ccc}0 & 0 & \lambda \\ 0 & 0 & -\lambda \\ \lambda & \lambda & 0\end{array}\right)$ for some $\lambda>0$. Then, $B\left(N_{1}^{0}, \dot{T}_{i}^{0} N_{p}^{0}\right)=-\lambda a_{1} c_{p}+\lambda a_{p} c_{1}>0$.

If $g_{1}^{0}$ and $g_{p}^{0}$ are asymptotic but their common end point is different from $(-1,0)$, we can arrange that this common end point is $(1,0)$. Then $N_{1}^{0}=$ $\left(a_{1}, b_{1}, c_{1}\right)$ and $N_{p}^{0}=\left(a_{p}, b_{p}, c_{p}\right)$ are both orthogonal to the line of $\mathbb{R}^{3}$ corresponding to $(1,0) \in \mathbb{R}^{2} \subset \mathbb{R P}^{2}$, and $a_{1}>0, b_{1}=a_{1}, c_{1}>0, a_{p}>0, b_{p}=a_{p}$, $c_{p}<0$. Then,

$$
B\left(N_{1}^{0}, \dot{T}_{i}^{0} N_{p}^{0}\right)=-\lambda a_{1} c_{p}-\lambda b_{1} c_{p}+\lambda a_{p} c_{1}+\lambda b_{p} c_{1}>0 .
$$

Finally, consider the case where $g_{1}^{0}$ and $g_{p}^{0}$ both have $(-1,0)$ as an end point. Note that this can happen only if $i=2$ and $p=3$. Then, $N_{1}^{0}=\left(a_{1}, b_{1}, c_{1}\right)$ and $N_{p}^{0}=\left(a_{p}, b_{p}, c_{p}\right)$ are both orthogonal to the line of $\mathbb{R}^{3}$ corresponding to $(-1,0) \in \mathbb{R}^{2} \subset \mathbb{R P}^{2}$, and $a_{1}>0, b_{1}=-a_{1}, c_{1}>0, a_{p}>0, b_{p}=-a_{p}, c_{p}<0$. Then,

$$
B\left(N_{1}^{0}, \dot{T}_{i}^{0} N_{p}^{0}\right)=-\lambda a_{1} c_{p}-\lambda b_{1} c_{p}+\lambda a_{p} c_{1}+\lambda b_{p} c_{1}=0 .
$$

BUlLETin DE LA SOCiÉtÉ MATHÉmATiQUe DE FRANCE 
Therefore, $B\left(N_{1}^{0}, \dot{T}_{i}^{0} N_{p}^{0}\right) \geq 0$ in all cases, with equality only in the last case above.

By choice of the indexing, the derivative $\dot{\ell}_{i}^{0}$ is non-negative for every $i$ with $2 \leq i \leq p-1$. We conclude that $B\left(N_{1}^{0}, \dot{N}_{p}^{0}\right) \geq 0$. Furthermore, the inequality is strict if at least one edge $e_{i}^{0}$ with $2 \leq i \leq p-1$ is labelled by + , unless $p=3$ and $e_{2}^{0}$ is horocyclic.

For a geometric interpretation of this inequality, note that $B\left(N_{1}^{0}, \dot{N}_{p}^{0}\right)$ is the derivative of $B\left(N_{1}^{t}, N_{p}^{t}\right)$ with respect to $t$ since we arranged that $N_{1}^{t}$ is constant, and that $B\left(N_{1}^{t}, N_{p}^{t}\right)$ is equal to cosh of the distance between the geodesics respectively containing $e_{1}^{t}$ and $e_{p}^{t}$.

Now, considering the other side of $\partial Q_{t}^{\text {Trun }}$, we see that

$$
\dot{N}_{p}^{0}=-\dot{\ell}_{1}^{0} \dot{T}_{1}^{0} N_{p}^{0}-\sum_{j=p+1}^{q} \dot{\ell}_{j}^{0} \dot{T}_{j}^{0} N_{p}^{0}
$$

The same argument as above shows that $B\left(N_{1}^{0}, \dot{T}_{j}^{0} N_{p}^{0}\right)<0$ for every $j$ with $p+1 \leq j \leq q$, unless $p+1=j=q$ and $e_{j}^{0}$ is horocyclic, in which case $B\left(N_{1}^{0}, \dot{T}_{j}^{0} N_{p}^{0}\right)=0$. Also,

$$
B\left(N_{1}^{0}, \dot{T}_{1}^{0} N_{p}^{0}\right)=-B\left(\dot{T}_{1}^{0} N_{1}^{0}, N_{p}^{0}\right)=-B\left(0, N_{p}^{0}\right)=0 .
$$

Since $\dot{\ell}_{j}^{0} \leq 0$ for every $p+1 \leq j \leq q$, we conclude that $B\left(N_{1}^{0}, \dot{N}_{p}^{0}\right) \leq 0$, and that the inequality is strict if at least one edge $e_{j}^{0}$ with $p+1 \leq j \leq q$ is labelled by - , unless $p+1=q$ and $e_{p+1}^{0}$ is horocyclic.

These inequalities can be reconciled only when one of the following holds:

(a) every edge $e_{i}^{0}$ with $i \neq 1, p$ is labelled by 0 ;

(b) $p=3$ and every edge $e_{i}^{0}$ with $p+1 \leq i \leq q$ is labelled by 0 ;

(c) $p=q-1$ and every edge $e_{i}^{0}$ with $2 \leq i \leq p-1$ is labelled by 0 .

If any edge $e_{i}^{0}$ was labelled by + or - , it would be possible to rechoose the indexing of the edges of $Q_{0}^{\text {Trun }}$ so Properties (a), (b) and (c) all fail, while the conditions imposed at the beginning of the proof still hold. (Use the fact that the untruncated polyhedron $Q_{0}$ has at least 3 edges.) As this would lead to a contradiction, we conclude that every edge of $Q_{0}^{\text {Trun }}$ is labelled by 0 .

Lemma 16. - For every face $Q$ of type (i) of the truncated polyhedron $P_{0}^{\text {Trun }}$, either all the edges of $\partial Q$ are labelled by 0 , or there are at least 4 sign changes in $\partial Q$.

Proof. - The face $Q$ corresponds to a face $Q^{\prime}$ of the projective polyhedron $P_{0}^{\text {Proj }}$. However, the vertices of $P_{x^{t}}$ associated to the vertices of $Q^{\prime}$ are not necessarily coplanar any more. Nevertheless, because the vector $\dot{x} \in T_{\Phi(P)}\left(\mathbb{R P}^{3}\right)^{V}$ tangent to $t \mapsto x^{t}$ is contained in the kernel of the tangent map $T_{\Phi(P)} \widetilde{\Theta}$, they are infinitesimally coplanar in the following sense: we can 
find a projective plane $\Pi_{t}$ in $\mathbb{R P}^{3}$ such that, for every vertex $v$ of $Q^{\prime}$, the distance from the vertex $x_{v}^{t}$ of $P_{x^{t}}$ corresponding to $v$ to the plane $\Pi_{t}$ is 0 and has derivative 0 at $t=0$, for an arbitrary riemannian metric on $\mathbb{R P}^{3}$. In addition, we can choose the plane $\Pi_{t}$ so that it depends differentiably on $t$. For instance, we can take $\Pi_{t}$ to be the plane passing through three predetermined such vertices of $P_{x^{t}}$.

For each vertex $v$ of $Q^{\prime}$, choose a point $y_{v}^{t} \in \Pi_{t}$, depending differentiably on $t$, such that $y_{v}^{0}=x_{v}^{0}$ and the distance from $y_{v}^{t}$ to $x_{v}^{t}$ has derivative 0 at $t=0$. In addition, we can choose $y_{v}^{t}$ so that it belongs to the sphere at infinity $\partial_{\infty} \mathbb{H}^{3}$ whenever $x_{v}^{t}$ does, namely whenever $x_{v}^{0}$ does by our choice of the curve $t \mapsto x^{t} \in$ $\left(\mathbb{R P}^{3}\right)^{V}$. Let $Q_{t}$ be the convex projective polygon in $\Pi_{t}$ whose vertices are the points $y_{v}^{t}$ associated to the vertices $v$ of $Q^{\prime}$, and which is uniquely determined by the property that it depends continuously on $t$ and that $Q_{0}=P_{x^{0}} \cap H_{0}$ (namely $Q_{0}$ is the face of $P^{\text {Proj }}=P_{x^{0}}$ corresponding to $Q^{\prime}$ ). Note that $Q_{t}$ is a hyperideal polygon in the projective plane $H_{t} \cong \mathbb{R P}^{2}$ with respect to the hyperbolic plane $H_{t} \cap \mathbb{H}^{3} \cong \mathbb{H}^{2}$, for $t$ sufficiently small.

In the hyperbolic plane $H_{t} \cap \mathbb{H}^{3}$, let $Q_{t}^{\text {Trun }}$ be the truncated polygon associated to the hyperideal polygon $Q_{t}$. Note that there is a natural identification between the vertices of $Q_{t}^{\text {Trun }}$ and those of the face $Q$ of $P_{0}^{\text {Trun }}$. Because the distance (for an arbitrary riemannian metric on $\mathbb{R P}^{3}$ ) from the vertex $y_{v}^{t}$ of $Q_{t}$ to the corresponding vertex $x_{v}^{t}$ of $P x_{t}$ is infinitesimally 0 , the hyperbolic distance between two vertices of $Q_{t}^{\text {Trun }}$ is infinitesimally equal to the hyperbolic distance between the corresponding vertices of $P_{x^{t}}^{\text {Trun }}$. In particular, if we label the edges of $Q_{0}^{\text {Trun }} \cong Q$ as above Lemma 13, the labelling is the same as that induced on the face $Q$ by our original labelling of $P_{0}^{\text {Trun }}$. Lemma 16 then follows from Lemma 15.

Finally, we consider faces of Type (ii) of $P^{\text {Trun }}$, contained in hyperbolic planes which are dual to strictly hyperideal vertices of $P^{\text {Proj }}$.

LEMmA 17. - In the hyperbolic plane $\mathbb{H}^{2}$, let $\left.Q_{t}, t \in\right]-\varepsilon, \varepsilon[$, be a differentiable family of compact strictly convex polygons with geodesic sides. Suppose that, at $t=0$, the derivative of the angle of $Q_{t}$ at each of its vertices is equal to 0 . Label each edge of $Q_{0}$ by the symbol,+ 0 or - according to whether the derivative of its length at $t=0$ is positive, zero or negative. Then, either all edges are labelled by 0 , or there are at least 4 sign changes in $\partial Q_{0}$.

Proof. - The proof is essentially the same as that of Lemma 15 (with no horocyclic sides). The reader familiar with [12] will also recognize here an infinitesimal (and simpler) version of [12, Lemma 4.11], which provided the inspiration for our proof of Lemma 15. 
Lemma 18. - For every face $Q$ of $P_{0}^{\text {Trun }}$ which corresponds to a face of type (ii) of the truncated polyhedron $P_{0}^{\text {Trun }}$, either all the edges of $\partial Q$ are labelled by 0 , or there are at least 4 sign changes in $\partial Q$.

Proof. - The proof is identical to that of Lemma 16, using Lemma 17 instead of Lemma 15.

We now conclude with a purely combinatorial argument.

LEMMA 19. - Label each edge of $P_{0}^{\text {Trun }}$ by a sign,+ 0 or - in such a way that, for each face $Q$ of $P_{0}^{\text {Trun }}$, either all the edges of $Q$ are labelled by 0 or there are at least 4 sign changes in $\partial Q$. Then, all the edges are labelled by 0.

Proof. — This is Cauchy's famous combinatorial lemma [5]; see also [15, Lemma T]. Since it is fairly simple, we include a proof for the sake of completeness.

Let $G$ be the 1 -skeleton of the dual cell decomposition of $\partial P_{0}^{\text {Trun }}$. Note that $G$ is a combinatorial graph, in the sense that an edge has two distinct end vertices and no two edges have the same end vertices. Label each edge of $G$ with the label of the dual edge of $P_{0}^{\text {Trun }}$. The sign condition for the faces of $\widehat{P}_{0}^{\text {Trun }}$ translates to a similar sign condition for each vertex $v$ of $G$ : either all the edges adjacent to $v$ are labelled by 0 , or one encounters at last 4 sign changes as one goes around $v$.

Lemma 19 then follows from the following statement.

Sublemma 20. - Let $G$ be a graph embedded in $\mathbb{S}^{2}$ with each edge of $G$ labelled by a sign,+ 0 or - in such a way that, for each vertex $v$ of $G$, either all the edges adjacent to $v$ are labelled by 0 , or one encounters at least 4 sign changes as we go around $v$. Then, all the edges of $G$ are labelled by 0 .

Proof of Sublemma 20. - Suppose that there is a graph $G$ for which the property fails. Erasing from $G$ all the edges labelled by 0 (which does not disturb the sign change condition) and restricting attention to a connected component of the remaining graph, we can assume that all edges are labelled by + or - , and that $G$ is connected and non-empty. Adding edges labelled by + if necessary (which again does not disturb the sign change condition), we can assume that the complement of $G$ in $\mathbb{S}^{2}$ consists of triangles.

For $i=0,2$, let $F_{i}$ be the number of triangles in the complement of $G$ with $i$ sign changes in their boundary. Then, if $V$ and $E$ respectively denote the number of vertices and edges of $G$, the hypothesis that there are at least 4 sign changes at each vertex implies that $4 V \leq 2 F_{2}$. Since we arranged that the complement of $G$ consists of triangles, counting edge sides in two different ways gives $3\left(F_{0}+F_{2}\right)=2 E$, while the Euler characteristic equation shows that $V-E+F_{0}+F_{2}=2$. Combining these three relations, we obtain that $F_{0}+4 \leq 0$, a contradiction. 
This completes the proof of Lemma 19

We are now ready to complete the proof of the Infinitesimal Rigidity Lemma of Proposition 10.

Proof of Proposition 10. - By Lemmas 14, 16 and 18, all the edges of $P_{0}^{\text {Trun }}$ are labelled by 0 . This means that if, for each edge $e$ of $P_{0}^{\text {Trun }}$, we consider the geodesic arc $e^{t}$ joining the vertices $v_{1}^{t}, v_{2}^{t}$ of $P_{x^{t}}^{\text {Trun }}$ corresponding to the end vertices $v_{1}, v_{2}$ of $e$, the length of $e^{t}$ has derivative 0 at $t=0$. In addition, if two edges $e_{1}$ and $e_{2}$ of $P_{0}^{\text {Trun }}$ meet at the vertex $v$, the angle between $e_{1}^{t}$ and $e_{2}^{t}$ at $v^{t}$ also has derivative 0 at $t=0$, because the tangent vector $\dot{x}=\mathrm{d} x^{t} / \mathrm{d} t_{\mid t=0}$ is contained in the kernel of the tangent map $T_{\Phi(P)} \widetilde{\Theta}$ (compare the polygons $Q_{t}$ which we used in the proofs of Lemmas 14, 16 and 18). Finally, we already saw in the proof of Lemma 16 that, for every face $Q$ of $P_{0}^{\text {Trun }}$ that corresponds to a face of $P_{0}^{\text {Proj }}$, the vertices of $P_{x^{t}}^{\text {Trun }}$ corresponding to the vertices of $Q$ are infinitesimally coplanar near $t=0$. If, among these faces $Q$ of $P_{0}^{\text {Trun }}$ corresponding to faces of $P_{0}^{\text {Proj }}$, we successively go from one to the other by crossing one edge at a time, we conclude that there exists an isometry $g_{t} \in \mathrm{PO}(3,1)$ of $\mathbb{H}^{3}$, depending differentiably on $t$ and with $g_{0}=\mathrm{Id}$, such that $\mathrm{d} g_{t}\left(v^{t}\right) / \mathrm{d} t_{\mid t=0}=0$ for every vertex $v^{t}$ of $P_{x^{t}}^{\text {Trun }}$ corresponding to a vertex $v$ of $P_{0}^{\text {Trun }}$.

Note that the vertices of the projective polyhedron $P_{x^{t}}$, namely the coordinates of $x^{t}$, can be completely recovered from the vertices of $P_{x^{t}}^{\text {Trun }}$ that correspond to vertices of $P_{0}^{\text {Trun }}$, as intersection of projective lines passing through these points. It follows that $\mathrm{d} g_{t}\left(x^{t}\right) / \mathrm{d} t_{\mid t=0}=0$. We conclude that

$$
\begin{aligned}
0=\mathrm{d} g_{t}\left(x^{t}\right) / \mathrm{d} t_{\mid t=0} & =\mathrm{d} g_{t}\left(x^{0}\right) / \mathrm{d} t_{\mid t=0}+\mathrm{d} x^{t} / \mathrm{d} t_{\mid t=0}, \\
& =\mathrm{d} g_{t}(\Phi(P)) / \mathrm{d} t_{\mid t=0}+\dot{x}
\end{aligned}
$$

since $g_{0}=\mathrm{Id}, x^{0}=\Phi(P)$, and $\mathrm{d} x^{t} / \mathrm{d} t_{\mid t=0}=\dot{x}$.

In particular, the vector $\dot{x}=-\mathrm{d} g_{t}(\Phi(P)) / \mathrm{d} t_{\mid t=0}=\mathrm{d} g_{t}^{-1}(\Phi(P)) / \mathrm{d} t_{\mid t=0}$ is tangent to the curve $t \mapsto g_{t}^{-1}(\Phi(P))$ at $t=0$. As a consequence, $\dot{x}$ is in the image of the tangent map at Id of the map $\mathrm{PO}(3,1) \rightarrow\left(\mathbb{R P}^{3}\right)^{V}$ defined by $g \mapsto g(\Phi(P))$.

This is exactly what we needed to complete the proof of Proposition 10.

\section{Convergence of hyperideal polyhedra}

We showed in Theorem 11 that the map $\Theta: \mathcal{P}_{\Gamma} \rightarrow K_{\Gamma} \subset \mathbb{R}^{E}$, which to a hyperideal polyhedron associates its dihedral angles, is a local homeomorphism. This section is devoted to proving that it is a covering map.

Recall that $K_{\Gamma}$ was defined as the set of those $\theta \in \mathbb{R}^{E}$ such that:

0 ) For every $e \in E$, the coordinate $\theta_{e}$ of $\theta$ corresponding to $e$ is in the interval $] 0, \pi[$; 
1) For every closed curve $\gamma$ embedded in $\Gamma$ and passing through the edges $e_{1}, e_{2}, \ldots, e_{n}$ of $\Gamma, \sum_{i=1}^{n} \theta_{e_{i}} \leq 2 \pi$ with equality allowed only when $\gamma$ is the boundary of a face of $\mathbb{S}^{2}-\Gamma$.

2) For every arc $\gamma$ embedded in $\Gamma$, passing through the edges $e_{1}, e_{2}, \ldots$, $e_{n}$ of $\Gamma$, and joining two distinct vertices which are in the closure of the same component of $\mathbb{S}^{2}-\Gamma$ but such that $\gamma$ is not contained in the boundary of that component, $\sum_{i=1}^{n} \theta_{e_{i}}<\pi$.

Proposition 21. - The map $\Theta: \mathcal{P}_{\Gamma} \rightarrow K_{\Gamma}$ is proper.

Proof. - Since $K_{\Gamma}$ is locally compact and $\mathcal{P}_{\Gamma}$ is metrizable, it suffices to prove the following: If $P_{n} \in \mathcal{P}_{\Gamma}, n \in \mathbb{N}$, is a sequence such that $\Theta\left(P_{n}\right)$ converges to some $\theta^{\infty} \in K_{\Gamma}$, then there is a subsequence $P_{n_{k}}, k \in \mathbb{N}$, which converges to a polyhedron $P_{\infty} \in \mathcal{P}_{\Gamma}$.

Consider such a sequence $P_{n} \in \mathcal{P}_{\Gamma}, n \in \mathbb{N}$. As usual, we use the same symbol $P_{n} \in \widetilde{\mathcal{P}}_{\Gamma}$ to denote a hyperideal polyhedron representing the class $P_{n} \in \mathcal{P}_{\Gamma}$, and we let $P_{n}^{\operatorname{Proj}}$ be the projective polyhedron associated to the polyhedron $P_{n}$. Let $x^{n}=\Phi\left(P_{n}\right) \in\left(\mathbb{R P}^{3}\right)^{V}$ be the vertex set of $P_{n}^{\text {Proj }}$. Pick three consecutive vertices $v_{1}, v_{2}, v_{3} \in V$ on the boundary of a face $f$ of the model polyhedron $P_{0}^{\text {Proj }}$. After modifying $P_{n}$ by an element of $\mathrm{PO}(3,1)$ if necessary, we can arrange that the corresponding vertices $x_{v_{1}}^{n}$ and $x_{v_{2}}^{n}$ of $P_{n}^{\text {Proj }}$ sit on the closure of the axis $\mathbb{R} \times\{0\} \subset \mathbb{R}^{3}$ in $\mathbb{R P}^{3}$ while the vertex $x_{v_{3}}^{n}$ is in the closure of the plane $\{0\} \times \mathbb{R}^{2}$ in $\mathbb{R P}^{3}$. By compactness of $\mathbb{R P}^{3}$, we can also assume after passing to a subsequence that $x^{n}$ converges to $x^{\infty} \in\left(\mathbb{R} \mathbb{P}^{3}\right)^{V}$.

For each face $f \in F$, the corresponding face $f^{n}$ of $P_{n}^{\text {Proj }}$ is contained in a projective plane $\Pi_{f}^{n}$ of $\mathbb{R P}^{3}$. After passing to a subsequence, we can assume that the plane $\Pi_{f}^{n}$ converges to a plane $\Pi_{f}^{\infty}$ for each $f \in F$. By construction, the limit plane $\Pi_{f}^{\infty}$ meets the closure of $\mathbb{H}^{3}$ in $\mathbb{R P}^{3}$. In particular, either it meets $\mathbb{H}^{3}$ or it is tangent to the sphere at infinity $\partial_{\infty} \mathbb{H}^{3}$.

Similarly, for an edge $e \in E$ joining two vertices $v, v^{\prime} \in V$, let $e^{n}$ be the

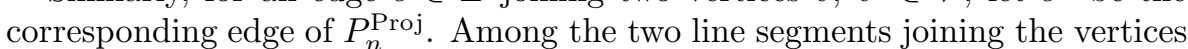
$x_{v}^{n}$ and $x_{v^{\prime}}^{n}$ of $P_{n}^{\text {Proj in }} \mathbb{R P}^{3}, e^{n}$ is also the one that meets $\mathbb{H}^{3}$. As $n$ tends to $\infty$, $e^{n}$ converges to a line segment $e^{\infty}$ which joins $x_{v}^{\infty}$ to $x_{v^{\prime}}^{\infty}$ and which meets the closure of $\mathbb{H}^{3}$ (after passing to a subsequence if by any chance $x_{v}^{\infty}=x_{v^{\prime}}^{\infty}$ ).

Note that the limit edge $e^{\infty}$ could conceivably be reduced to a single point. We first prove that this does not happen.

LEMmA 22. - There is no edge $e \in E$ such that the corresponding limit edge $e^{\infty}$ is reduced to a single point.

Proof of Lemma 22. - Suppose otherwise, and that there is a point $p \in \mathbb{R P}^{3}$ such that $E_{p}=\left\{e \in E ; e^{\infty}=p\right\}$ is non-empty. Note that $p$ must be on the sphere at infinity $\partial_{\infty} \mathbb{H}^{3}$. Indeed, it must be in the closure of $\mathbb{H}^{3}$ since each $e^{n}$ meets $\mathbb{H}^{3}$, but cannot be in $\mathbb{H}^{3}$ since the end points of $e^{n}$ are outside $\mathbb{H}^{3}$.

TOME $130-2002-\mathrm{N}^{\mathrm{O}} 3$ 
Consider the vertices $v_{1}, v_{2}$ and $v_{3}$ which we selected at the beginning of the proof of Proposition 21. The edge $e_{12}$ joining $v_{1}$ to $v_{2}$ cannot be in $E_{p}$, since the corresponding line segment $e_{12}^{\infty}$ contains the diameter $\mathbb{R} \times\{0\} \cap \mathbb{H}^{3}$. The edge $e_{23}$ joining $v_{2}$ to $v_{3}$ cannot be in $E_{p}$ either, because its end points $x_{v_{2}}^{\infty}$ and $x_{v_{3}}^{\infty}$ are distinct since they are respectively contained in the closures of $\mathbb{R} \times\{0\}$ and $\{0\} \times \mathbb{R}^{2}$, which are disjoint. In the boundary of the model polyhedron $P_{0}^{\text {Proj }}$, let $K_{p}$ be the union of all the edges $e \in E_{p}$. By the above remark and because $e_{12}$ and $e_{23}$ are contained in the same face of $P_{0}^{\text {Proj }}$, the interiors of $e_{12}$ and $e_{23}$ are contained in the same component of $\partial P_{0}^{\text {Proj }}-K_{p}$. Let $U$ be a regular neighborhood of $K_{p}$ in $\partial P_{0}^{\text {Proj }}$, and let $\gamma$ be a component of $\partial U$ which is contained in the same component of $\partial P_{0}^{\text {Proj }}-K_{p}$ as the interiors of $e_{12}$ and $e_{23}$.

Let $e_{1}, e_{2}, \ldots, e_{m}, e_{m+1}=e_{1} \in E$ be the edges of $P_{0}^{\text {Proj }}$ traversed by $\gamma$, in this order. Note that $\gamma$ defines a closed curve $\gamma_{\Gamma}$ in the dual graph $\Gamma$, crossing the edges corresponding to $e_{1}, e_{2}, \ldots, e_{m}$. This closed curve is not necessarily embedded, but it is immersed in $\Gamma$ in the sense that each $e_{i}$ is different from $e_{i+1}$. Also, by construction, each limit edge $e_{i}^{\infty}$ has one end point equal to $p$, but is not reduced to this point.

Let $f_{1}, f_{2}, \ldots, f_{m}, f_{m+1}=f_{1}$ be the faces met by $\gamma$, so that $\gamma$ crosses the edge $e_{i}$ between the faces $f_{i}$ and $f_{i+1}$. By construction, each of the limit planes $\Pi_{f_{i}}^{\infty}$ passes through $p$. In $\mathbb{R}^{3}$, the euclidean plane $\Pi_{f_{i}}^{\infty} \cap \mathbb{R}^{3}$ bounds a preferred euclidean half-space $H_{f_{i}}^{\infty}$, namely the limit of the half space $H_{f_{i}}^{n}$ bounded by $\Pi_{f_{i}}^{n} \cap \mathbb{R}^{3}$ and containing the polyhedron $P_{n} \subset \mathbb{H}^{3}$. The intersection $C$ of these half-spaces $H_{f_{i}}^{\infty}$ is a convex cone with vertex $p$, containing the limit edges $e_{i}^{\infty}$ in its boundary. In addition, every extremal half-line of $C$ must coincide with one of the $e_{i}^{\infty}$ near $p$.

The intersection of $C$ with a small horosphere $S \subset \mathbb{H}^{3}$ centered at $p$ is a polygon $Q_{S}$, possibly non-compact and/or reduced to a subset of a geodesic of $S$. Note that the polygon $Q_{S}$ is convex for the euclidean metric of $S$ induced by the hyperbolic metric of $\mathbb{H}^{3}$.

We split the analysis into several cases.

Case 1. None of the limit edges $e_{i}^{\infty}$ is tangent to $\partial_{\infty} \mathbb{H}^{3}$.

In particular, the planes $\Pi_{f_{i}}^{\infty}$ all meet $\mathbb{H}^{3}$ and define hyperbolic planes $\mathbb{H}^{3} \cap \Pi_{f_{i}}^{\infty}$. As $n$ tends to $\infty$, the hyperbolic plane $\mathbb{H}^{3} \cap \Pi_{f_{i}}^{n}$ converges to $\mathbb{H}^{3} \cap \Pi_{f_{i}}^{\infty}$. The dihedral angle between two such consecutive planes $\mathbb{H}^{3} \cap \Pi_{f_{i}}^{n}$ and $\mathbb{H}^{3} \cap \Pi_{f_{i+1}}^{n}$ is equal to the coordinate $\theta_{e_{i}}^{n}$ of $\theta^{n}=\Theta\left(P_{n}\right) \in \mathbb{R}^{E}$ corresponding to $e_{i} \in E$. Because $\theta^{n}$ converges to a point $\left.\theta^{\infty} \in K_{\Gamma} \subset\right] 0, \pi\left[{ }^{E}\right.$, the limit $\theta_{e_{i}}^{\infty}$ of $\theta_{e_{i}}^{n}$ is different from 0 and $\pi$. It follows that the two hyperbolic planes $\mathbb{H}^{3} \cap \Pi_{f_{i}}^{n}$ and $\mathbb{H}^{3} \cap \Pi_{f_{i+1}}^{n}$ have non-trivial intersection, and that their dihedral angles along this intersection geodesic is equal to $\theta_{e_{i}}^{\infty}$. Note that the hyperbolic dihedral angle between the hyperbolic planes $\mathbb{H}^{3} \cap \Pi_{f_{i}}^{n}$ and $\mathbb{H}^{3} \cap \Pi_{f_{i+1}}^{n}$ is also equal to the euclidean 
angle between the two lines $S \cap \Pi_{f_{i}}^{n}$ and $S \cap \Pi_{f_{i+1}}^{n}$ in the horosphere $S$. It follows that the polygon $Q_{S}=C \cap S$ is compact and, as in the proof of Proposition 5, that $\sum_{i=1}^{m} \theta_{e_{i}}^{\infty}=2 \pi$.

Because of the hypothesis that $\theta^{\infty}$ satisfies Condition 1 in the definition of $K_{\Gamma}$, this implies that the closed curve $\gamma_{\Gamma}$ in $\Gamma$ defined by $\gamma$ is embedded, and bounds a component of $\mathbb{S}^{2}-\Gamma$ corresponding to a vertex $v_{4} \in V$. It follows that a component $W$ of $\partial P_{0}^{\text {Proj }}-\gamma$ contains only one vertex of $P_{0}^{\text {Proj }}$, namely $v_{4}$. This $W$ cannot be a component of the interior of the regular neighborhood $U$ of $K_{p}$ since, by definition of $K_{p}$, each of these components contains at least one edge of $P_{0}^{\text {Proj }}$. Therefore, $W$ must be a component of $\partial P_{0}^{\text {Proj }}-U$ and meets the interiors of the edges $e_{12}$ and $e_{23}$. As a consequence, the vertex $v_{4}$ in $W$ must be equal to $v_{2}$, and $v_{1}$ and $v_{3}$ must be in $K_{p}$. However, this would imply that $x_{v_{1}}^{\infty}=x_{v_{3}}^{\infty}=p$, contradicting the fact that $x_{v_{1}}^{\infty}$ and $x_{v_{3}}^{\infty}$ belong to disjoint subsets of $\mathbb{R P}^{3}$, namely the respective closures of $\mathbb{R} \times\{0\}$ and $\{0\} \times \mathbb{R}^{2}$. We consequently reach a contradiction in the case where none of the limit edges $e_{i}^{\infty}$ is tangent to $\partial_{\infty} \mathbb{H}^{3}$.

Case 2. Some but not all limit edges $e_{i}^{\infty}$ are tangent to $\partial_{\infty} \mathbb{H}^{3}$, and all those $e_{i}^{\infty}$ which are tangent to $\partial_{\infty} \mathbb{H}^{3}$ are equal.

Assume that $e_{i}^{\infty}=e_{i+1}^{\infty}=\cdots=e_{j}^{\infty}$ are tangent to $\partial_{\infty} \mathbb{H}^{3}$ but that $e_{i-1}^{\infty}$ and $e_{j+1}^{\infty}$ are both different from $e_{i}^{\infty}=e_{j}^{\infty}$. Then, for $i<k \leq j$, the plane $\Pi_{f_{k}}^{\infty}$ may be tangent to $\partial_{\infty} \mathbb{H}^{3}$ and in particular have trivial intersection with $\mathbb{H}^{3}$. As a consequence, we loose any geometric control on the angles $\theta_{e_{k}}^{n}$ with $i \leq k \leq j$.

However, by hypothesis, the two edges $e_{i-1}^{\infty}$ and $e_{j+1}^{\infty}$ cannot be tangent to $\partial_{\infty} \mathbb{H}^{3}$. It follows that the two planes $\Pi_{f_{i}}^{\infty}$ and $\Pi_{f_{j+1}}^{\infty}$ intersect the horosphere $S$ in two geodesic lines $\Pi_{f_{i}}^{\infty} \cap S$ and $\Pi_{f_{j+1}}^{\infty} \cap S$. These two geodesic lines are parallel since $\Pi_{f_{i}}^{\infty}$ and $\Pi_{f_{j+1}}^{\infty}$ meet along the line containing $e_{i}^{\infty}$, which is disjoint from $\mathbb{H}^{3}$. In addition, $\Pi_{f_{i}}^{\infty} \cap S$ and $\Pi_{f_{j+1}}^{\infty} \cap S$ delimit an end of the polygon $Q_{S}=S \cap C$ which is adjacent to the tangent limit edge $e_{i}^{\infty}$.

The convex polygon $Q_{S}$ can have no other end. Indeed, the only possibility for this would be that the convex cone $C$ locally contains near $p$ a line tangent to $\partial_{\infty} \mathbb{H}^{3}$, contradicting our hypothesis that all those $e_{i}^{\infty}$ which are tangent to $\partial_{\infty} \mathbb{H}^{3}$ are tangent to each other. We conclude that $e_{k}^{\infty}$ is tangent to $\partial_{\infty} \mathbb{H}^{3}$ exactly when $i \leq k \leq j$. Considering the dihedral angles at the corners of $\partial Q_{S}$ as in Case 1, it now follows that $\sum_{k=j+1}^{i-1} \theta_{e_{j}}^{\infty}=\pi$, counting indices modulo $m$.

In the graph $\Gamma$, let $\gamma_{\Gamma}^{\prime}$ be the part of the immersed curve $\gamma_{\Gamma}$ which crosses the edges $e_{j+1}, e_{j+2}, \ldots, e_{i-1}$. Because $\sum_{k=j+1}^{i-1} \theta_{e_{j}}^{\infty}=\pi$ and because $\theta^{\infty}$ satisfies Condition 1 in the definition of $K_{\Gamma}$, the $\operatorname{arc} \gamma_{\Gamma}^{\prime}$ is actually embedded in $\Gamma$.

For $i \leq k<j$, the limit edges $e_{k}^{\infty}$ and $e_{k+1}^{\infty}$ are equal and tangent to $\partial_{\infty} \mathbb{H}^{3}$ at $p$. If we orient the edges $e_{k}$ and $e_{k+1}$ by the outer normal orientation of $\partial U$ along $\gamma$, we conclude that they must end on the same vertex $v$ of $P_{0}^{\text {Proj }}$. (Otherwise, an edge of the face $f_{k+1}^{n}$ of $P_{n}^{\text {Proj }}$ would be unable to meet $\mathbb{H}^{3}$ ).

TOME $130-2002-\mathrm{N}^{\mathrm{O}} 3$ 
As a consequence, the faces $f_{i}$ and $f_{j+1}$ share this vertex $v \in V$. In particular, the end points of $\gamma_{\Gamma}^{\prime}$ (namely the vertices of $\Gamma$ respectively corresponding to $f_{i}$ and $\left.f_{j+1}\right)$ are contained in the closure of the same component $A$ of $\mathbb{S}^{2}-\Gamma$, namely the component corresponding to $v \in V$. Since $\theta^{\infty}$ satisfies Condition 2 in the definition of $K_{\Gamma}$ and since $\sum_{k=j+1}^{i-1} \theta_{e_{j}}^{\infty}=\pi$, we conclude that $\gamma_{\Gamma}^{\prime}$ is contained in the boundary of $A$. But this implies that the closed curve $\gamma_{\Gamma}$ is equal to the boundary of the component $A$ of $\mathbb{S}^{2}-\Gamma$. We already saw in Case 1 that this is impossible, as this leads to a contradiction.

Case 3. Some but not all limit edges $e_{i}^{\infty}$ are tangent to $\partial_{\infty} \mathbb{H}^{3}$, and at least two of the $e_{i}^{\infty}$ which are tangent to $\partial_{\infty} \mathbb{H}^{3}$ are different.

The convex polygon $Q_{S}=S \cap C$ in the horosphere $S$ is non-compact since some $e_{i}^{\infty}$ is tangent to $\partial_{\infty} \mathbb{H}^{3}$. On the other hand, the hypothesis that there exists an $e_{j}^{\infty}$ which is not tangent to $\partial_{\infty} \mathbb{H}^{3}$ provides a corner of $\partial Q_{S}$ where the external dihedral angle is at least $\theta_{e_{j}}^{\infty}>0$. It follows that the boundary $\partial Q_{S}$ is non-empty and connected. There consequently exists indices $i_{1}, i_{2}$ such that the limit edge $e_{i}^{\infty}$ is tangent to $\partial_{\infty} \mathbb{H}^{3}$ if and only if $i_{1} \leq i \leq i_{2}$, counting indices modulo $m$.

If $e_{i}^{\infty}$ and $e_{i+1}^{\infty}$ are tangent to $\partial_{\infty} \mathbb{H}^{3}$, either $e_{i}^{\infty}=e_{i+1}^{\infty}$, or $e_{i}^{\infty}$ and $e_{i+1}^{\infty}$ are collinear and point in opposite directions at $p$. Indeed, this immediately follows by consideration of the possible limits for the face $f_{i+1}^{n}$, using the fact that it is a convex polygon and that each of its edges must meet $\mathbb{H}^{3}$. (There are two cases, according to whether $\Pi_{f_{i+1}}^{\infty}$ is tangent to $\partial_{\infty} \mathbb{H}^{3}$ or not). If $e_{i}^{\infty}$ and $e_{i+1}^{\infty}$ pointed in opposite directions at $p$, this would provide a geodesic line which is completely contained in the convex polyhedron $Q_{S}$, which is incompatible with the fact that $\partial Q_{S}$ has a corner with strictly positive external dihedral angle. Therefore $e_{i}^{\infty}=e_{i+1}^{\infty}$ for all $i_{1} \leq i<i_{2}$. But this contradicts the hypothesis that there are at least two different $e_{i}^{\infty}$ which are tangent to $\partial_{\infty} \mathbb{H}^{3}$.

Case 4. All the limit edges $e_{i}^{\infty}$ are tangent to $\partial_{\infty} \mathbb{H}^{3}$.

In particular, the boundary $\partial Q_{S}$ has no corners, and consists of 0,1 or 2 parallel geodesic lines. As a consequence, there can be at most two indices $i$ such that $\Pi_{f_{i}}^{\infty}$ is not tangent to $\partial_{\infty} \mathbb{H}^{3}$.

For each $i$, either $e_{i}^{\infty}=e_{i+1}^{\infty}$, or $e_{i}^{\infty}$ and $e_{i+1}^{\infty}$ are collinear and point in opposite directions at $p$; in addition, if we orient the edges $e_{i}$ and $e_{i+1}$ by the outer normal orientation of $\partial U$ along $\gamma$, their terminal end points must necessarily be equal in the first case, and distinct in the second case. Again, this follows by consideration of the limit of the face $f_{i+1}^{n}$, using the fact that it is a convex polygon and that each of its edges must meet $\mathbb{H}^{3}$. Note that only the second alternative can hold if $\Pi_{f_{i+1}}^{\infty}$ is not tangent to $\partial_{\infty} \mathbb{H}^{3}$.

If all the limit edges $e_{i}^{\infty}$ are equal, then the edges $e_{i}$ all have a vertex $v$ in common, and $\gamma$ just turns around this vertex $v$. However, as in Case 1 , this contradicts the fact that the interiors of the two edges $e_{12}$ and $e_{23}$ are contained in the same component of $\partial P_{0}^{\text {Proj }}-K_{p}$ as $\gamma$. 
Otherwise, counting indices modulo $m$, there are $i, j$ such that $e_{i}^{\infty}=e_{i+1}^{\infty}=$ $\cdots=e_{j}^{\infty}$ but such that $e_{i-1}^{\infty}$ and $e_{j+1}^{\infty}$ are both different from $e_{i}^{\infty}=e_{j}^{\infty}$. Then, the oriented edges $e_{k}$, with $i \leq k \leq j$ have the same terminal end point $v$, which is distinct from the terminal end point $v^{\prime}$ of $e_{i-1}$ and from the terminal end point $v^{\prime \prime}$ of $e_{j+1}$. The two vertices $v^{\prime}$ and $v^{\prime \prime}$ are actually equal; otherwise, one would get a contradiction from the limit of the convex triangle $T_{n}$ in $P_{n}^{\text {Proj }}$ whose vertices are the vertices of $P_{n}^{\text {Proj }}$ corresponding to $v, v^{\prime}$ and $v^{\prime \prime}$, using the fact that by Lemma 3 each edge of $T_{n}$ must meet $\mathbb{H}^{3}$.

We conclude from this analysis that the terminal end point of each $e_{i}$ with $1 \leq i \leq m$ must be equal to $v$ or $v^{\prime}=v^{\prime \prime}$.

If $v$ and $v^{\prime}$ are joined by an edge $e \in E$, then $\gamma$ must be the boundary of a regular neighborhood of $e$, by 3 -connectedness of $\Gamma$. In particular, the edge $e_{12}$ must be equal to $e$ or to one of the $e_{i}$ with $1 \leq i \leq m$, by definition of $\gamma$. However, the limit edge $e^{\infty}$ joins the limit points $x_{v}^{\infty}$ and $x_{v^{\prime}}^{\infty}$, and is contained in a line tangent to $\partial_{\infty} \mathbb{H}^{3}$ at $p$. Since $e_{12}^{\infty}$ contains the diameter $\mathbb{R} \times\{0\} \cap \mathbb{H}^{3}$, it follows that $e_{12}$ cannot be equal to $e$. Similarly, $e_{12}$ cannot be equal to one of the $e_{i}$ as $e_{i}^{\infty}$ is tangent to $\partial_{\infty} \mathbb{H}^{3}$ at $p$ by definition of $\gamma$.

Finally, if $v$ and $v^{\prime}$ are not joined by an edge, then the $e_{i}$ are the only edges whose interior is contained in the same component of $\partial P_{0}^{\text {Proj }}-K_{p}$ as $\gamma$. This again contradicts the definition of $\gamma$ since $e_{12}$ cannot be equal to any of these $e_{i}$, as above.

As a consequence, we reach a contradiction in all cases. This completes the proof of Lemma 22 .

LEMMA 23. - No plane $\Pi_{f}^{\infty}$ is tangent to $\partial_{\infty} \mathbb{H}^{3}$.

Proof of Lemma 23. - Suppose that $\Pi_{f}^{\infty}$ is tangent to $\partial_{\infty} \mathbb{H}^{3}$. Then, because no limit edge $e^{\infty}$ is reduced to a single point, by Lemma 22, the consideration of the possible degenerations of the face $f^{n}$ of $P_{n}^{\text {Proj }}$ shows the following: There are two edges $e_{1}$ and $e_{2}$ of the face $f$, meeting at a vertex $v$, such that the limit point $x_{v}^{\infty}$ is on the sphere $\partial_{\infty} \mathbb{H}^{3}$ and such that the limit edges $e_{1}^{\infty}$ and $e_{2}^{\infty}$ point in opposite directions at $x_{v}^{\infty}$.

By consideration of the convex cone $C$ delimited by the limit planes $\Pi_{f^{\prime}}^{\infty}$ associated to the faces $f^{\prime} \in F$ that contain $v$, one concludes as in the proof of Lemma 22 that there exists a third edge $e_{3}$ containing $v$ such that $e_{3}^{\infty}$ is equal to $e_{1}^{\infty}$ and $e_{2}^{\infty}$. However, this provides a face $f_{0}$, containing $v$, such that the convex polygon $f_{0}^{n}$ converges to a line segment that is tangent to $\partial_{\infty} \mathbb{H}^{3}$ at one of its end points. This is not possible if no limit edge $e^{\infty}$ is reduced to a point.

This proves Lemma 23.

For an edge $e \in E$, we already know by Lemma 22 that the limit edge $e^{\infty}$ is not reduced to a single point. If $f$ and $f^{\prime} \in F$ are the two faces separated by $e$, Lemma 23 shows that the hyperbolic planes $\Pi_{f}^{\infty} \cap \mathbb{H}^{3}$ and $\Pi_{f^{\prime}}^{\infty} \cap \mathbb{H}^{3}$ are

TOME $130-2002-\mathrm{N}^{\mathrm{O}} 3$ 
non-trivial. Since the dihedral angle $\theta_{e}^{n}$ between the hyperbolic planes $\Pi_{f}^{n} \cap \mathbb{H}^{3}$ and $\Pi_{f^{\prime}}^{n} \cap \mathbb{H}^{3}$ converges to the coordinate $\theta_{e}^{\infty} \neq 0, \pi$ of the limit point $\theta^{\infty} \in K_{\Gamma}$, it follows that $\Pi_{f}^{\infty} \cap \mathbb{H}^{3}$ and $\Pi_{f^{\prime}}^{\infty} \cap \mathbb{H}^{3}$ have a non-trivial intersection, and make dihedral angle of $\theta_{e}^{\infty}$ along this intersection. In particular, the limit edge $e^{\infty}$ meets $\mathbb{H}^{3}$.

For a face $f \in F$, the plane $\Pi_{f}^{n}$ containing the corresponding face $f^{n}$ of $P_{n}^{\text {Proj }}$ converges to a plane $\Pi_{f}^{\infty}$ which meets $\mathbb{H}^{3}$ and, for each edge $e$ of $f$, the edge $e^{n}$ of $f^{n}$ converges to an edge $e^{\infty}$ which meets $\mathbb{H}^{3}$ but whose end points are outside of $\mathbb{H}^{3}$. It follows that $f^{n}$ converges to a convex polygon $f^{\infty} \subset \Pi_{f}^{\infty}$ which is really 2-dimensional and has the same combinatorics as $f$.

As a consequence, as $f$ ranges over all the elements of $F$, the union of the polygons $f_{\infty}$ forms a polyhedral sphere $S_{\infty}$ which has the same combinatorial type as $\partial P_{0}^{\text {Proj. }}$. In addition, $S_{\infty}$ is locally convex since the dihedral angle $\theta_{e}^{\infty}$ of $S_{\infty}$ along the edge $e^{\infty}$ corresponding to $e \in E$ is in $] 0, \pi[$.

Let $P_{\infty}^{\text {Proj }} \subset \mathbb{R P}^{3}$ be bounded by the sphere $S_{\infty}$, oriented by its identification

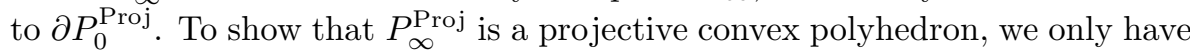
to show that it does not contain any projective line. (Remember that we had included this condition in the definition of convex polyhedra in $\left.\mathbb{R P}^{3}\right)$. However, by local convexity, the only way this could fail is if $P_{\infty}^{\text {Proj }}$ was projectively equivalent to an infinite prism in $\mathbb{R}^{3} \subset \mathbb{R} \mathbb{P}^{3}$, in which case the dual graph of the cell decomposition of $\partial P_{\infty}^{\text {Proj }}=S_{\infty}$ would be homeomorphic to a circle. But this dual graph is $\Gamma$ by construction, and cannot be a circle by 3 -connectedness.

Therefore, $P_{\infty}^{\text {Proj }}$ is a convex projective polyhedron. We already noted that its vertices are all outside $\mathbb{H}^{3}$, and that its edges all meet $\mathbb{H}^{3}$. Therefore $P_{\infty}=P_{\infty}^{\text {Proj }} \cap \mathbb{H}^{3}$ is a hyperideal polyhedron. By construction, the vertices of $P_{\infty}^{\text {Proj }}$ are the limit of the vertices of $P_{n}^{\text {Proj }}$, and it follows that $P_{\infty}$ is the limit of the sequence $P_{n}$ in $\mathcal{P}_{\Gamma}$.

This concludes the proof of Proposition 21.

If we retrace back the proof of Proposition 21, and in particular the proof of Lemma 22, we can summarize it as follows: If the sequence $P_{n} \in \mathcal{P}_{\Gamma}, n \in \mathbb{N}$, admits no converging subsequence then, possibly after passing to a subsequence, it necessarily degenerates according to one of the following patterns:

1) one of the dihedral angles of $P_{n}$ converges to 0 or $\pi$;

2) $P_{n}$ develops a very long and thin 'neck' around a very short simple closed curve in $\partial P_{n}$ which corresponds to a fixed curve in the model polyhedron $\partial P_{0}$;

$3)$ the truncated polyhedron $P_{n}^{\text {Trun }}$ develops a very long and thin 'neck' around a very short simple closed curve in $\partial P_{n}^{\text {Trun }}$ which corresponds to a fixed curve in $\partial P_{0}^{\text {Trun }}$ and crosses exactly one of the new faces of $\partial P_{n}^{\text {Trun }}$ associated to vertices of $P_{0}^{\text {Proj }}$.

An immediate corollary of Proposition 21 is the following. 
Corollary 24. - The map $\Theta: \mathcal{P}_{\Gamma} \rightarrow K_{\Gamma}$ is a covering map.

Proof. - The map $\Theta$ is a local homeomorphism by Theorem 11, and it is proper by Proposition 21. Since $K_{\Gamma}$ is locally compact, this implies that $\Theta$ is a covering map.

\section{Realizing large angles}

To realize hyperideal polyhedra with large external dihedral angles, namely with small internal dihedral angles, we will rely on Andreev's classification of compact hyperbolic polyhedra with acute internal dihedral angles [2]. We first state this classification with our current terminology.

Recall that the complete graph $K_{n}$ is the graph with $n$ vertices where any pair of distinct vertices are joined by one edge. Let $K_{n}^{-}$be obtained from $K_{n}$ by removing one arbitrary edge.

THEOREM 25 (see [2]). — Let $\Gamma$ be a planar 3-connected graph, different from the graphs $K_{4}$ and $K_{5}^{-}$. Let a weight $\theta_{e} \in\left[\frac{1}{2} \pi, \pi[\right.$ be attached to each edge e of $\Gamma$. There exists a compact polyhedron $P$ in $\mathbb{H}^{3}$ with dual graph isomorphic to $\Gamma$ and with external dihedral angle $\theta_{e}$ at the edge corresponding to the edge e of $\Gamma$ if and only if the following three conditions are satisfied:

1) every component of $\mathbb{S}^{2}-\Gamma$ contains exactly three edges $e_{1}, e_{2}$ and $e_{3}$ of $\Gamma$, and $\sum_{i=1}^{3} \theta_{e_{i}}<2 \pi$;

2) for every simple closed curve $\gamma$ embedded in $\Gamma$ and passing through exactly three edges $e_{1}, e_{2}$ and $e_{3}, \sum_{i=1}^{3} \theta_{e_{i}}>2 \pi$ unless $\gamma$ is the boundary of a component of $\mathbb{S}^{2}-\Gamma$;

3) for every simple closed curve $\gamma$ embedded in $\Gamma$ and passing through exactly four edges $e_{1}, e_{2}, e_{3}$ and $e_{4}, \sum_{i=1}^{4} \theta_{e_{i}}>2 \pi$ unless $\gamma$ bounds in $\mathbb{S}^{2}$ a diamond made up of two components of $\mathbb{S}^{2}-\Gamma$ and of one edge of $\Gamma$ separating them.

In addition, if $P$ exists, it is unique up to isometry of $\mathbb{H}^{3}$.

Andreev also provides a similar result for the graph $K_{5}^{-}$, for which there is an additional condition. Note that $K_{5}^{-}$is the dual graph of a prism with triangular basis. The case of the graph $K_{4}$, which is the dual graph of a tetrahedron, is treated in [17]. However, we will not need to consider these two graphs.

Consider a strictly hyperideal polyhedron $P_{0} \in \mathcal{P}_{\Gamma}$, and its associated truncated polyhedron $P_{0}^{\text {Trun }}$. The dual graph $\Gamma^{\text {Trun }}$ of $P_{0}^{\text {Trun }}$ is obtained from $\Gamma$ as follows: For each component $A$ of $\mathbb{S}^{2}-\Gamma$, add to $\Gamma$ a vertex $v_{A}$ and join by an edge this new vertex $v_{A}$ to each vertex of $\Gamma$ located in the boundary of $A$. In particular, $\Gamma^{\text {Trun }}$ can be described purely in terms of $\Gamma$. Also, note that each edge of $\Gamma^{\text {Trun }}-\Gamma$ corresponds to an edge of $P_{0}^{\text {Trun }}$ where the external dihedral angle is equal to $\frac{1}{2} \pi$.

TOME $130-2002-\mathrm{N}^{\mathrm{O}} 3$ 
Proposition 26. - Let $\Gamma$ be a planar 3 -connected graph with at least 4 vertices, and with a weight $\left.\theta_{e} \in\right] \frac{2}{3} \pi, \pi[$ attached to each edge $e$ of $\Gamma$. Then there exists a strictly hyperideal polyhedron $P$ in $\mathbb{H}^{3}$ with dual graph isomorphic to $\Gamma$ and with external dihedral angle $\theta_{e}$ at the edge corresponding to the edge e of $\Gamma$. In addition, $P$ is unique up to isometry of $\mathbb{H}^{3}$.

Proof. - Let $\Gamma^{\text {Trun }}$ be the graph associated to $\Gamma$ as above. Let us associate to each edge $e$ of $\Gamma^{\text {Trun }}$ the weight $\theta_{e} \in\left[\frac{2}{3} \pi, \pi[\right.$ of the data of Proposition 26 if $e$ is in $\Gamma \subset \Gamma^{\text {Trun }}$, and the weight $\theta_{e}=\frac{1}{2} \pi$ otherwise. We first show that $\Gamma^{\text {Trun }}$ with these edge weights satisfy the conditions of Theorem 25.

By construction, $\Gamma^{\text {Trun }}$ is planar. One easily checks that it is 3-connected. Also, $\Gamma$ has at least 4 vertices and $\mathbb{S}^{2}-\Gamma$ has at least 4 components, by 3 connectedness. It follows that $\Gamma^{\text {Trun }}$ has at least 8 vertices, and in particular is neither $K_{4}$ nor $K_{5}^{-}$.

Every component of $\mathbb{S}^{2}-\Gamma^{\text {Trun }}$ is a triangle bounded by two edges $e_{1}$ and $e_{2}$ of $\Gamma^{\text {Trun }}-\Gamma$ and one edge $e_{3}$ of $\Gamma$. Then $\theta_{e_{1}}=\theta_{e_{2}}=\frac{1}{2} \pi$ and $\theta_{e_{3}}<\pi$, so that $\sum_{i=1}^{3} \theta_{e_{i}}<2 \pi$. As a consequence, Condition 1 of Theorem 25 is satisfied.

Let $\gamma$ be a simple closed curve embedded in $\Gamma^{\text {Trun }}$ which crosses exactly three edges $e_{1}, e_{2}$ and $e_{3}$. If at least one vertex of $\gamma$ is in $\Gamma^{\text {Trun }}-\Gamma$, it easily follows from the 3 -connectedness of $\Gamma$ that $\gamma$ is the boundary of a component of $\mathbb{S}^{2}-\Gamma^{\text {Trun }}$. Otherwise, each $e_{i}$ is also an edge of $\Gamma$ because the edges of $\Gamma^{\text {Trun }}-\Gamma$ all join a vertex of $\Gamma$ to a vertex of $\Gamma^{\text {Trun }}-\Gamma$. It follows that each $\theta_{e_{i}}$ is greater than $\frac{2}{3} \pi$, and therefore that $\sum_{i=1}^{3} \theta_{e_{i}}>2 \pi$. Therefore, Condition 2 of Theorem 25 is satisfied.

Finally, let $\gamma$ be a simple closed curve embedded in $\Gamma^{\text {Trun }}$ which crosses exactly four edges $e_{1}, e_{2}, e_{3}$ and $e_{4}$. It can contain at most two vertices of $\Gamma^{\text {Trun }}-\Gamma$, since no two vertices of $\Gamma^{\text {Trun }}-\Gamma$ are adjacent in $\Gamma^{\text {Trun }}$. If $\gamma$ contains two vertices of $\Gamma^{\text {Trun }}-\Gamma$, it again follows from the 3-connectedness of $\Gamma$ that $\gamma$ bounds a diamond made up of the union of two components of $\mathbb{S}^{2}-\Gamma^{\text {Trun }}$ and of one edge of $\Gamma$. If $\gamma$ contains exactly one vertex of $\Gamma^{\text {Trun }}-\Gamma$, say the vertex separating $e_{1}$ from $e_{2}$, then $e_{1}$ and $e_{2}$ are both in $\Gamma^{\text {Trun }}-\Gamma$ and $e_{3}$ and $e_{4}$ are edges of $\Gamma$, so that $\theta_{e_{1}}=\theta_{e_{2}}=\frac{1}{2} \pi, \theta_{e_{3}}>\frac{2}{3} \pi$ and $\theta_{e_{4}}>\frac{2}{3} \pi$; it follows that $\sum_{i=1}^{4} \theta_{e_{i}}>\frac{7}{3} \pi>2 \pi$. Finally, if no vertex of $\gamma$ is in $\Gamma^{\text {Trun }}-\Gamma$, then each $e_{i}$ is in $\Gamma$, so that $\theta_{e_{i}}>\frac{2}{3} \pi$; consequently $\sum_{i=1}^{4} \theta_{e_{i}}>\frac{8}{3} \pi>2 \pi$ in this case. It follows that Condition 3 of Theorem 25 is satisfied.

By Theorem 25, there consequently exists a compact polyhedron $P^{\prime}$ in $\mathbb{H}^{3}$ whose dual graph is isomorphic to $\Gamma^{\text {Trun }}$ and with external dihedral angle $\theta_{e}$ at the edge corresponding to the edge $e$ of $\Gamma^{\text {Trun }}$.

The faces of $P^{\prime}$ correspond to vertices of $\Gamma^{\text {Trun }}$, and therefore are of two types: those which correspond to vertices of $\Gamma$, and those which correspond to vertices of $\Gamma^{\text {Trun }}-\Gamma$. Let $f_{1}, f_{2}, \ldots, f_{n}$ be the faces of $P^{\prime}$ that correspond to vertices of $\Gamma$, and let $\Pi_{i} \subset \mathbb{R P}^{3}$ be the projective plane containing $f_{i}$. Let $P^{\text {Proj }}$ be the closure of the component of $\mathbb{R P}^{3}-\bigcup_{i=1}^{n} \Pi_{i}$ that contains the interior of $P^{\prime}$.

BULLETIN DE LA SOCIÉtÉ MATHÉMATIQUE DE FRANCE 
At this point, we know that the dual graph of the cell decomposition of $\partial P^{\text {Proj }}$ contains $\Gamma$ and has no additional vertex. If $f$ is a face of $P^{\prime}$ which corresponds to a vertex of $\Gamma^{\text {Trun }}-\Gamma$, namely to a component of $\mathbb{S}^{2}-\Gamma$, and if $f_{i_{1}}, f_{i_{2}}, \ldots, f_{i_{k}}$ are the faces of $P^{\prime}$ adjacent to $f$, the fact that the $f_{i_{j}}$ are orthogonal to $f$ implies that the projective planes $\Pi_{i_{j}}$ all pass through the point $\Pi^{\perp} \in \mathbb{R P}^{3}-\mathbb{H}^{3} \cup \partial_{\infty} \mathbb{H}^{3}$ dual to the projective plane $\Pi$ containing $f$. It follows that the dual graph of $\partial P^{\text {Proj }}$ has no additional edges, and is equal to $\Gamma$.

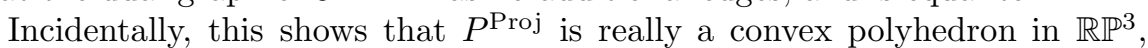
namely satisfies the additional condition that it contains no projective line. Otherwise, it would be projectively equivalent to the closure of an infinite prism in $\mathbb{R}^{3} \subset \mathbb{R P}^{3}$ and its dual graph $\Gamma$ would be homeomorphic to a circle, which is excluded by 3 -connectedness.

This also shows that the hyperbolic polyhedron $P=P^{\operatorname{Proj}} \cap \mathbb{H}^{3}$ is strictly hyperideal. By construction of $P^{\prime}$, the external dihedral angle of $P$ along the edge corresponding to the edge $e$ of $\Gamma$ is equal to $\theta_{e}$.

Conversely, the uniqueness of $P$ follows from the uniqueness of $P^{\prime}$ provided by Theorem 25 .

\section{Proof of Theorems 1 and 2}

We are now ready to complete the proof of Theorems 1 and 2, by considering again the map $\Theta: \mathcal{P}_{\Gamma} \rightarrow K_{\Gamma} \subset \mathbb{R}^{E}$, which to a hyperideal polyhedron associates its dihedral angles. Indeed, by definition of $K_{\Gamma}$, Theorem 1 is equivalent to the property that $\Theta$ is surjective, whereas Theorem 2 is equivalent to the fact that $\Theta$ is injective. Both statements are simultaneously provided by the following result.

Proposition 27. - The map $\Theta: \mathcal{P}_{\Gamma} \rightarrow K_{\Gamma}$ is a homeomorphism.

Proof. - We proved in Corollary 24 that $\Theta: \mathcal{P}_{\Gamma} \rightarrow K_{\Gamma}$ is a covering map. By definition, $K_{\Gamma}$ is convex in $\mathbb{R}^{E}$, and in particular it is simply connected. It follows that the covering is trivial. Now, Proposition 26 shows that, for every $\theta \in] \frac{2}{3} \pi, \pi\left[{ }^{E}, \Theta^{-1}(\theta)\right.$ consists of exactly one point. This proves that the covering map $\Theta: \mathcal{P}_{\Gamma} \rightarrow K_{\Gamma}$ is actually a homeomorphism.

\section{BIBLIOGRAPHY}

[1] Alexandrow (A.D.) - Konvexe Polyeder, Akademie-Verlag, Berlin, 1958.

[2] Andreev (E.M.) - On Convex Polyhedra in Lobačevskiu Spaces (Russian), Mat. Sbornik, t. 81 (123) (1970), pp. 445-478; English transl., Math. USSR Sb. t. 10 (1970), pp. 413-440.

TOME $130-2002-\mathrm{N}^{\mathrm{O}} 3$ 
[3] _ On Convex Polyhedra of Finite Volume in Lobačevskiน Spaces (Russian), Mat. Sbornik, t. 83 (125) (1970), pp. 256-260; English transl., Math. USSR Sb. t. 12 (1970), pp. 255-259.

[4] Bao (X.) - Hyperideal Polyhedra in Hyperbolic 3-Space, Doctoral dissertation, University of Southern California, Los Angeles, 1998.

[5] Cauchy (A.) - Sur les polygones et les polyèdres, J. École Polytechnique, t. 9 (1813), pp. 87-98.

[6] Coxeter (H.S.M.) - On Complexes with Transitive Groups of Automorphisms, Ann. of Math., t. 35 (1934), pp. 588-621.

[7] Cromwell (P.R.) - Polyhedra, Cambridge University Press, 1997.

[8] Grünbaum (B.) - Convex Polytopes, Interscience Publishers, John Wiley \& Sons Inc., New York, 1967.

[9] VAn Lint (J.H.) \& Wilson (R.M.) - A Course in Combinatorics, Cambridge University Press, 1992.

[10] Rivin (I.) - Euclidean Structures on Simplicial Surfaces and Hyperbolic Volume, Ann. of Math., t. 139 (1994), pp. 553-580.

[11] A Characterization of Ideal Polyhedra in Hyperbolic 3-Space, Ann. of Math., t. 143 (1996), pp. 51-70.

[12] Rivin (I.) \& Hodgson (C.D.) - A Characterization of Compact Convex Polyhedra in Hyperbolic 3-Space, Invent. Math., t. 111 (1993), pp. 77-111; Corrigendum, Invent. Math. t. 117 (1994), pp. 359.

[13] SChlenker (J.-M.) - Métriques sur les polyèdres hyperboliques convexes, J. Diff. Geom., t. 48 (1998), pp. 323-405.

[14] _ Dihedral Angles of Convex Polyhedra, Discrete Comp. Geom., t. 23 (2000), pp. 409-417.

[15] Stoker (J.J.) - Geometrical Problems Concerning Polyhedra in the Large, Comm. Pure Appl. Math., t. 21 (1958), pp. 119-168.

[16] Thurston (W.P.) - Three-Dimensional Geometry and Topology, (S. Levy, ed.), Princeton Math. Series, vol. 35, Princeton University Press, 1997.

[17] VinBerg (亡̀.B.) - Discrete groups generated by reflections in Lobačevskiı spaces, Mat. Sbornik, t. 72 (114) (1967), pp. 471-488; English transl., Math. USSR Sb. t. 1 (1967), pp. 429-444; Correction, Mat. Sbornik t. 73 (115) (1967), pp. 303. 